(Physiologisches Laboratorium in Bonn.)

\title{
Ueber den Einfluss der umgebenden Temperatur auf den Stoffwechsel der Warmblüter.
}

\author{
Vòn \\ Dr. Giuseppe Colasanti aus Rom.
}

(Nebst Tafel II.)

Dass die Warmblïter die Temperatur im Inneren ihres Körpers - d. h. der grossen Körperhöhlen - trotz grosser Variation der Temperatur der Umgebung constant zu erhalten vermögen, ist zum Theil durch diejenigen compensatorischen Vorrichtungen des Thierkörpers bedingt, welche den $\mathrm{W}$ ärmeverlus t reguliren. Hierüber herrscht unter den Gelehrten keine Meinungsverschiedenheit. Zahlreiche Forscher haben aber theils Thatsachen dafür beigebracht, theils ihre Veberzengung anf Grund ihrer Beobachtungen dahin ausgesprochen, dass die warmblütigen Thiere und der Mensch auch durch eine Anpassung der Wärmeproduction an den Wärmeverlust die Temperatur ihres Körper-Inneren innerhalb gewisser Grenzen constant zu erhalten vermögen. Unter diesen Forschern mögen nur Crawford, Lavoisier und Seguin, Delaroche, Regnault und Reiset, Vierordt, Letellier, Lehmann, Liebermeister, Gildemeister, Ludwig und Sanders-Ezn, Röhrig und Z Zuntz, Pflüger und Andere hervorgehoben werden. Gleichwohl ist die Frage über die durch veränderte Wärmeproduction vermittelte Wärmeregulation durchaus controvers.

Es lässt sich allerdings nicht in Abrede stellen, dass bei den älteren Forschern Bedenken erhoben werden können, die auf den damaligen ungenauen 'gasometrischen Methoden beruhen. Regnault und Reiset haben sich bei ihren Untersuchungen keineswegs das Ziel gesetzt gehabt, auch diese Frage in den Bereich ihrer Forschungen zu zichen. Die Aufgabe wäre sonst längst definitiv gelöst, da 
Einfluss d. umgebenden Temperatur auf d. Stoffwechsel d. Warmblüter. 93

bessere und zuverlässigere Versuche im Gebiete der Respiration niemals ausgeführt worden sind. Nur beiläufig sind also von Regnault und Reiset einige Beobachtungen gemacht worden, welche in hohem Grade für die Steigerung der Wärmeproduction bei $\mathrm{Ab}$ kühlung der Thiere sprechen.

Gegen die meisten anderen Versuche (Vierordt, Letellier, Liebermeister, Gildemeister) lässt sich das Bedenken erheben, welches aus der Nichtberücksichtigung des Sauerstoffverbrauches entspringt. Denn diese Untersuchungen stützen ihre Argumentation nur auf die Kohlensäure, deren Exhalation fast immer nur für sehr kurze Zeit beobachtet worden ist. Da nun die in dem Körper angehäuften (absorbirten, locker und fest gebundenen) Kohlensäuremassen ganz ausserordentlich gross sein können, in Wirklichkeit aber unbekannt sind, so bedingt nothwendig jede Veränderung der Strömungsgeschwindigkeit des Blutes durch die Lungen, jede Veränderung der Athemmechanik eine an Grösse nicht sicher bekannte Veränderung in der Grösse der Kohlensäureexhalation, ohne dass darum auf eine Veränderung der Kohlensäureproduction geschlossen werden kann - Verhältnisse, die schon Pflüger an verschiedenen Stellen umständlich dargelegt hat.

Dass ferner bei den Versuchen von Ludwig und SandersEzn am Respirationsapparate undichte Stellen waren, hat Pflüger aus den Zahlen dieser Forscher bewiesen.

Bei den von Röhrig-Zuntz und Pflüger im Bonner physiologischen Laboratorium ausgeführten Untersuchungen ist Sauerstoff und Kohlensäure gemessen und der Beweis geliefert, dass die Energie der respiratorischen Functionen (chemisch genommen) mit abnehmender Temperatur der Umgebung bei Warmblütern wächst. Die Zahl and zeitliche Dauer dieser Versuche war aber nicht gross genug, um den Einwand vollkommen zu entkräften, dass diese Variation der Wärmeproduction nur in den ersten kurzen Zeiten nach stattgehabtem Temperaturwechsel in der Umgebung der Thiere anhalte.

Auch war vor Anstellung neuer Versuche erst die Frage über den Einfluss der Athemmechanik auf den Stoffwechsel zu erledigen, die von Pflüger, Finkler und Oertmann in neuester Zeit entschieden worden ist.

Die calorimetrischen Methoden haben wegen der ihnen heute noch anhaftenden Unsicherheit die Controverse über die Wärmeregulation ebenfalls noch nicht zum Abschlusse zu bringen vermocht. 
Bei der theoretischen und praktischen Wichtigkeit der Frage forderte mich deshalb Prof. Pflüger auf, die Wärmeregulation vom Gesichtspunkte der Variation der Wärmeproduction unter seiner Leitung einer eingehenden Untersuchung zu unterwerfen. Da die Energie der Oxydationsprocesse des thierischen Organismus heute mit hinreichender Schärfe unzweideutig bestimmt werden kann, so sollte hierauf das Augenmerk gerichtet werden.

Diese Untersuchung wurde von mir mit Hülfe eines von Prof. P fl üg er construirten Respirationsapparates ausgeführt, zu dessen Beschreibung ich sofort übergehe.

\section{A. Respirationsapparat.}

Der Respirationsapparat ist dazu bestimmt, den Gaswechsel kleinerer Thiere quantitativ zu bestimmen. Derselbe ist im Wesentlichen gebaut nach dem Gedanken, den zuerst Lavoisier gehabt hat und den dann Regnault und Reiset in so eleganter Form ausgeführt haben. So vortrefflich auch immer und unübertroffen der Regnaul t'sche Apparat dasteht, so unterliegt es doch keinem Zweifel, dass er mit zwei wesentlichen Uebelständen behaftet ist, die bei unserem Apparate vollkommen vermieden worden sind. Der erste Uebelstand besteht darin, dass das Gas des Respirationsraumes in nicht hinreichenden Contact mit der Kalilauge der Aspiratoren gebracht wird; daher kommt es denn, dass Regnault und Reiset bei länger dauernden Versuchen immer einen sehr hohen Kohlensäuregehalt der Luft fanden, die das Thier athmen musste. Pflüger hat demnach Quecksilberaspiratoren hergestellt, welche die Luft durch Kalilauge treiben, und es auf diese Weise erreicht, dass sogar am Ende länger dauernder Versuche die aus dem Recipienten, in dem das Thier ist, angesogene Luft nur einige Zehntel Procent Kohlensäure enthält.

Der zweite Uebelstand des Regnault'schen Apparates besteht in den ausserordentlich langen Gummischläuchen, die an sehr vielen Stellen durch Bindfaden mit den betreffenden Theilen des Respirationsapparates in luftdichte Verbindung gesetzt werden müssen. Es ist nun allerdings nicht in Abrede zu stellen, dass besonders bei Anwendung starker Druckdifferenzen zwischen dem Inneren und der Umgebung des Gummirohres eine geringe Diffusion stattfindet, wie das schon von Graham nachgewiesen ist. Bei der Kleinheit dieses 
Gasaustausches und den bei den Respirationsversuchen in der That immer nur sehr geringen Druckdifferenzen, und den verhältnissmässig sehr bedeutenden Grössen für. Sa uerstoffverbrauch und Kohlensäureentwicklung, kommt jener Fehler ganz gewiss kaum in Betracht. Nach den Erfahrungen, die in dem Bonner physiologischen Laboratorium gemacht worden sind, liegt die Gefahr bei der Anwendung der Gummischläuche vielmehr in schmalen ventilartig wirkenden Gängen, welche sich an fast allen Gummischläuchen finden und in der Art der Fabrikation dẹselben ihre Ursache haben. Da nun das Gummi je nach der Temperatur eine verschiedene Consistenz besitzt, und die einzelnen Theilchen des Gummis hierbei ihre gegenseitige Lage ändern, so kommt es vor, dass ein Schlauch, der durch Saugen oder Druck bei bestimmter Temperatur selbst viele Stunden lang nach Aussage des Manometers als vollkommen luftdicht sich erwiesen hat, gleich darauf undicht wird, wenn die Temperatur gestiegen ist, oder wenn die Consistenz des Gummis durch Bewegung des Schlauches sich verändert hat. Derselbe Schlauch ist nach einigen Stunden nach Abnahme der Temperatur wieder dicht. Dies ist ein Uebelstand, gegen den die grösste Sorgfalt und Gewissenhaftigkeit in der Herstellung des Apparates keine vollständige Gewährleistung erzielt.

Da nun die bekannte Stickstoffexhalation, welche Regnault und Reiset bei ihren Versuchen sehr allgemein beobachtet haben, heute fast allgemein auf Undichtigkeit des Regnault'schen Apparates bezogen wird, obwohl in diesem immer Ueberdruck vorhanden war, da ausserdem bei den im Bonner Laboratorium ausgeführten Untersuchungen von Herrn Hugo Schulz, der mit einem kleinen modificirten Regnault'schen Apparat gearbeitet hat, ebenfalls gewöhnlich eine. scheinbare Stickstoffexhalation beobachtet ist, so war die Aufgabe gestellt, solche Bedingungen an dem Apparate herzustellen, welche die Diffusion zwischen Gummi und atmosphärischer Luft vollkommen ausschliessen. Diese Bedingungen wurden erreicht einmal dadurch, dass der Gummischlauch da, wo er nicht entbehrt werden konnte, unter Wasser versenkt wurde. Diejenigen Röhrenleitungen, welche durch die Luft gehen mussten, bestanden aus dickwandigen, auf ihre Luftdichtheit wohl geprüften Bleirohren. Diese Bleirohre communicirten mit dem Recipienten, in dem das Thier sich befindet, durch gelöthete Verbindungsstellen und tauchten unter Wasser mit demjenigen Ende, auf welches Gummischläuche 
aufgebunden waren. An denjenigen Orten, wo dennoch an den durch die Luft gehenden Rohrleitungen ein Gummirohrverband eingeschaltet werden musste, benutzten wir zur vollkommenen Sicherung des Verschlusses eine Vorrichtung, die wir als Wassermanschette (s. Taf. II. $x$ ) bezeichnen wollen, und welche folgende Einrichtung hat: die Gummirohrverbindung ist in diesem Falle noch umschlossen von einem weiteren Glasrohr, welches an seinem Ende durch ein aufgebundenes Gummirohr auf die Röhrenleitung aufgebunden und ausserdem mit Wasser erfüllt ist.

Es befindet sich nun an dem Regnault'schen Apparate noch eine Stelle, die Veranlassung zur Undichtigkeit sein kann. Das ist nämlich die Klappe, welche im Fussboden des Thierrecipienten angebracht ist und durch welche das Thier in die Athemglocke geführt wird. Dieser Theil des Apparates, also der Thierrecipient, steht bei uns in einer Zinkwanne $(Q)$, welche soweit $\left(\omega_{2}\right)$ mit Wasser gefüllt wird, dass sämmtliche Theile der Klappe unter Wasser sind, welches sofort in das Innere des Apparates eindringen muss und bei unserer Einrichtung sichtbar wird, sobald die Klappe nicht absolut sicher schliesst. Das sind die allgemeinen wesentlichen Abweichungen von der Regnault'schen Vorrichtung; wir gehen nunmehr zu der specielleren Beschreibung der einzelnen Theile des Respirationsapparates über.

Der Thierrecipient (siehe Tafél II.) besteht aus einer Messingplatte, auf welche die Glasglocke 0 aufgekittet ist. Dieselbe hat bei uns einen Inhalt von genau 3400 Cubikcentimeter. In diese Glocke $O$ führt also von unten her durch die horizontale Metallplatte ein rundes Loch, welches durch eine Klappe luftdicht verschliessbar ist. Umgeben ist diese Glocke von dem Glascylinder $P$, der ebenfalls auf die Metallplatte hermetisch aufgekittet ist und dazu dient, das Wasser aufzunehmen, welches die Glasglocke 0 von aussen vollkommen umgibt. Das Wasserniveau ist bei $\omega, \omega$ (s. Fig.). Die Glocke $O$ trägt auf ihrer Kuppel einen luftdicht aufgekitteten metallischen Aufsatz $\delta$, der von fünf eingelötheten Metallröhren durchbohrt wird. In die eine dieser Metallröhren ist ein Thermometer ( $\varrho)$ eingekittet, welches weit in den Raum der Glocke hineinragt. Damit das Quecksilbergefäss nicht mit dem Thier in Berührung komme, ist dasselbe mit einer vielfach durchlöcherten Metallhülse (N) umgeben, befindet sich übrigens so hoch an der Decke der Glocke, dass die Thiere unter der Glocke auch mit dieser Metallhülse selbst 
niemals in unmittelbare Berührung kommen können. Die Scala dieses Thermometers ragt hoch über den Aufsatz $\delta$, ja sogar den Wasserspiegel im Cylinder $\mathrm{P}$ empor. Auf diese Weise glaubten wir sicherer die Temperatur zu erhalten, welche unter der Glocke wirklich ist, wenn sich das Thier darunter befindet. Regnault pflegte bekanntlich sich zu begnügen mit der Messung der Temperatur in dem Wasser des Cylinders P. Aus dem Binnenraum der Glocke führt nun das Metallrohr L nach dem mit ihm luftdicht verbundenen 1 Centimeter weiten Glasrohr $M$, welches in einen mit concentrirter Chlorcalciumlösung gefüllten Glascylinder taucht. Die Vorrichtung M ist dázu bestimmt, als Manometer zu dienen, weil wir die Absicht hatten, abweichend von Regnault, den Druck im Inneren des Respirationsraumes immer gleich dem atmosphärischen zu erhalten. Die Empfindlichkeit eines solchen Manometers dem Quecksilbermanometer gegenüber bedarf ja keiner Begründung. Was nun die Vorrichtungen betrifft, durch welche die von dem Thiere producirte Kohlensäure aus dem Athemraume fortgeschafft wurde, so dienten dazu die beiden graduirten Aspiratoren $Z_{1}, Z_{2}$ und die mit Kalilauge gefüllten Müller'schen Ventile $\mathrm{S}_{1} \mathrm{~S}_{2}, \mathrm{~T}_{1} \mathrm{~T}_{2}$. Wenn der eine Aspirator Luft aus dem Respirationsranme $O$ saugt, wobei die Luft durch die Kalilauge streichen muss, treibt der andere Aspirator ebensoviel Luft durch die Kalilauge zurück nach dem Respirationsraum 0 .

Specieller war nun die Einrichtung der Aspiratoren folgende: Auf einer Console, die in die Wand gemauert war, standen zwei grosse starke Gläser $\mathrm{Y}_{1}$ und $\mathrm{Y}_{2}$. Diese Gläser waren mit Quecksilber gefüllt and von oben her tauchten die Glascylinder $Z_{1} Z_{2}$, die überall vollkommen geschlossen waren, mit ihren unteren Oeffnungen in das Quecksilber, so dass der Raum in dem Aspirator Z durch Glas und Quecksilber vollständig hermetisch abgeschlossen war. Um diesen Aspirationsraum Z nun mit den Athemräumen in hermetische Communication setzen zu können, war das S-förmig gebogene Glasrohr $X_{1}$ und $X_{2}$ auf dem Boden der Standgläser $Y_{1}$ und $\mathrm{Y}_{2}$ mit Gyps festgekittet, so zwar, dass durch die Mitte der Quecksilberoberfläche das 'freie offene Ende dieses \$-förmig gebogenen Glasrohres in den Aspirationsraum $\mathrm{Z}$ hineinragte. Diese Glasrohre $\mathrm{X}_{1} \quad \mathrm{X}_{2}$ setzen sich nun continuirlich ohne Einschaltung von Gummi oder Kittung fort bis unter das Wasser der Wanne W, wo sie mit den Gummischläuchen der Ventile also 
unter Wasser in hermetische Communication gesetzt werden. Die Leitungen von beiden Aspiratoren zu der Respirationsglocke $O$ sind vollkommen symmetrisch und vollkommen congruent construirt. Dies ist in der Zeichnung nicht wiedergegeben, weil es hier darauf an$\mathrm{kam}$, die Leitungen so verlaufen zu lassen, dass der Leser einen möglichst klaren Einblick in die Natur des Mechanismus erhält. Die auf- und niedergehende Bewegung der Aspiratoren $Z_{1} Z_{2}$ wurde in einfacher Weise durch Gelenkverbindungen bewirkt, welche zu dem Balancier $(\alpha)$ gehen, der in seiner Mitte auf dem Stativ $(\gamma)$ ruht. An dem einen Arme des Balanciers greift die periodisch aufund niedergehende Triebstange $(\beta)$ an, welche nach dem Zapfen der Scheibe eines Uhrwerks führt, das durch ein schweres Gewicht in Bewegung gesetzt wird. Um die Geschwindigkeit des Ganges dieses Uhrwerkes reguliren zu können, ist an demselben eine Windflïgelvorrichtung angebracht, deren Flügelstellung das Gewünschte innerhalb weiter Grenzen leistet. In der Zeichnung ist das Uhrwerk weggelassen. Die mit Kalilauge gefüllten Müll er'schen Ventile bestehen aus einem einzigen Stück Glas, an dessen Rohre sich die Gummischläuche ansetzen. Die in der Zeichnung angebrachten Pfeile geben die Richtung an, in welcher das Ventil die Bewegung des Gases gestattet. Betrachten wir demnach beispielsweise den $\mathrm{Cy}$ linder $Z_{2}$ während er aufsteigt, so ist es klar, dass ihm die Luft zuströmt durch die Leitung $\mathrm{X}_{2} \mathrm{~S}_{2} \mathrm{R}_{2} \mathrm{~J}$ von dem Respirationsraum 0 . Der Verlust, den der Respirationsraum $O$ erleidet, wird wieder ersetzt durch Leitung $\mathrm{K} \mathrm{T}_{1} \mathrm{X}_{1} \mathrm{Z}_{1}$. Wenn umgekehrt $\mathrm{Z}_{2}$ niedersteigt, entweicht aus ihm die Luft durch $\mathrm{X}_{2}, \mathrm{~T}_{2} \mathrm{~J}$, geht durch $\mathrm{O}$ nach $\mathrm{K}$, durch $R_{1}$ nach $S_{1} X_{1} Z_{1}$. Damit nun diese sämmtlichen Ventile $\mathrm{S}_{1} \mathrm{~S}_{2}, \mathrm{~T}_{1} \mathrm{~T}_{2}$ mit Einschluss der Gummischläuche und der Verbindung der Gummischläuche mit den Bleiröhren $\mathrm{J} K$ und den Glasröhren $\mathrm{X}_{1}{ }^{~} \mathrm{X}_{2}$ von jeder Communication mit atmosphärischer Luft abgeschlossen seien, sind sie untergetaucht in die vollkommen mit Wasser bis $\omega_{3} \omega_{3}$ gefüllte Wanne W. Um die lufthaltigen Ventile zu zwingen, unter dem Wasser zu bleiben, und immer vertikal zu stehen, ist ein schwerer dicker eiserner Ring $U_{1}$ und $U_{2}$ um das untere Abflussröhrchen gelegt und durch um die oberen Glasröhren der M üller'schen Ventile geschlungene Drähte jedes Ventil an Stäben vertical aufgehangen, die über dem Wasser in der Luft quer über dem Rande der Wanne $\mathrm{W}$ befestigt sind. Diese sind in der Zeichnung weggelassen und die Ventile freischwebend gezeichnet. Um 
Einfluss d. umgebenden Temperatur auf d. Stoffwechsel d. Warmbiüter. $9 \ddot{9}$

nun zu jeder Zeit, oder am Ende des Versuches die Zusammensetzung des Gases unter der Athemglocke 0 wissen zu können, sind eingeschaltet in das Röhrensystem der Wanne W die beiden Glaskugeln $\mathrm{R}_{1} \mathrm{R}_{2}$.

Wie man aus der Zeichnung ersieht, geht durch diese Glaskugeln der durch die Aspiration bewegte Luftstrom in immer derselben Richtung, d. h. er geht von dem Raum, in dem das Thier ist, zu den Kaliventilen; hat also seine Kohlensäure noch nicht abgegeben. Um die Luft zu zwingen, immer durch diese Kugeln $R_{1}$ $R_{2}$ zu gehen, ist während des Versuches die zu der Kugel $R_{1} R_{2}$ angebrachte Nebenschliessung $\sigma_{1} \sigma_{2}$ durch eine Klemme geschlossen. Soll nun z. B. die Luft der Kugel $R_{1}$ analysirt werden, wird die Nebenschliessung $\sigma_{1}$ geöffnet und darauf werden diesseits und jenseits $R_{1}$ die Gummischläuche abgeklemmt mit 4 hermetisch schliessenden Klemmen bei $\mu \mu \nu \nu$. Nachdem diese natürlich immer unter Wasser angelegt sind, wobei eine Veränderung der Temperatur der Kugel $R_{1}$ möglichst vermieden wird, werden die Gummischläuche auf jeder Seite der Kugel $R_{1}$ zwischen $\mu$ und $\nu$ durchschnitten und diese in der Bunsen'schen Gaswanne in ein grosses Absorptionsrohr entleert. Es ist klar, dass hierbei der Versuch nicht die leiseste Störung erfährt, die Luft in dem Athemraum und seinen Annexen nicht die geringste Aenderung ihrer Temperatur, ihres Druckes und ihrer procentischen Zusammensetzung.

In der vorliegenden Zeichnung sieht man nur vier Ventile; wir hatten deren aber in Wirklichkeit doppelt so viel in der Wanne W. Die andern vier, welche weggelassen sind, haben genau dieselbe Anordnung wie diejenigen, welche sich in der Zeichnung finden. Bei jedem einzelnen Versuche arbeiten gleichzeitig auch nur vier Ventile, die anderen vier sind unthätig, um in einem gegebenen Augenblick durch geeignete Oeffnung und Schliessung von Klemmen nunmehr an die Reihe zu kommen, während die anderen ausgeschaltet werden. Auf diese Weise hat man es in der Hand, die Kohlensäureausgabe in kleineren Zeiträumen controliren zu können, ein Vortheil, der sehr hoch anzuschlagen und bei der Regnault'schen Anordnung nicht vorhanden ist. Es braucht kaum bemerkt zu werden, dass die Röhrchen $v_{1} \cdot v_{2}$ mit ihren Hähnen zum Zulassen und Ablassen der Kalilauge in und aus den Mülle r'schen. Ventilen dienen, die Röhrchen $\xi_{1} \xi_{2}$ aber, um den Binnenraum des Müller'schen Ventils mit der äusseren Luft in Communication setzen zu 
können durch Oeffnen des Hahnes $\xi_{1} \xi_{2}$, wobei natürlich das Müller'sche Veatil aus dem Wasser gehoben worden ist.

Es ist wohl kaum nöthig hervorzuheben, dass vor dem Beginn eines neuen Versuches und vor Füllung der Kaliventile nach Entfernung der Aspiratoren $Z_{1} Z_{2}$ ein kräftiger Luftstrom mit einem Blasebalg bei geschlossener Klappe des Thierrecipienten durch den ganzen Raum getrieben wurde.

Es erubrigt uns nun die Beschreibung der Vorrichtung, durch welche der von dem Thier verbrauchte Sauerstoff der Athemglocke 0 zugeführt wird. Der 1700 Cc. haltende Glasballon C, der vollkommen mit starker Chlorcalciumlösung gefüllt ist, wird, während Hahn $\mathrm{E}$ geschlossen, Hahn $\mathrm{F}$ und Hahn $\mathrm{D}$ geöffnet ist und Leitung $\varphi$ mit dem Sauerstoffgasometer in Verbindung steht, durch Ausfliessen der Chlorcalciumlösung durch Hahn $\zeta$ mit Sauerstoff gefüllt, und der Sauerstoff im Ballon C unter Atmosphärendruck gestellt durch Regulation der Wasserhöhe in Rohr B. Nachdem im Zimmer eine constante Temperatur sich hergestellt hat, und abermals genau durch Regulation des Wasserstandes im Druckrohr B das Gas in $\mathrm{C}$ unter Atmosphärendruck gestellt ist, wird Temperatur und Barometerstand abgelesen, so dass das in C befindliche Sauerstoffquantum genau bekannt ist. Die Röhrenleitung $\varphi$ bleibt immer mit dem Sauerstoffgasometer in Verbindung, Hahn D, E und $\mathbf{F}$ werden natürlich nach der Füllung von $\mathrm{C}$ mit Sauerstoff geschlossen. In die Röhrenleitung $(\varphi)$ ist ein T-rohr eingeschaltet, dessen einer Schenkel $(\lambda)$ an seinem Ende hakenförmig gekrümmt und mit einem Geissler'schen Hahne versehen ist. Diese Seitenleitung hat den Zweck, jedesmal wenn der Ballon $\mathrm{C}$ frisch gefüllt wird, und zwar fast zu gleicher Zeit, aus dem Gasometer eine Sauerstoffprobe zu entnehmen, damit dieselbe auf ihre Reinheit gasometrisch untersucht werden kann. Das Gläschen $G$ stellt ein mit concentrirter Chlorcalciumlösung gefülltes Ventilfläschchen vor, durch welches der Sauerstoff aus $\mathrm{C}$ durch $\mathrm{H}$ nach $\mathrm{O}$ geht, wenn der Versuch im Gange ist, wobei selbstverständlich Hahn $\mathrm{E}$ und $\mathrm{F}$ offen sind, während D geschlossen ist. Vor dem Versuch ist diesseits des Hahnes E Sauerstoff, jenseits atmosphärische Luft. An der Stelle, wo Rohr $\mathrm{H}$ in den Thierrecipienten einmündet, sollte ein Hahn sein, was bei der Herstellung dieses Apparates herzustellen leider vergessen worden ist. Da wir darauf verzichteten, wie Regnault mit Ueberdruck zu arbeiten, und doch immer ein sachverständiger Beobachter zweck- 
Einfluss d. umgebenden Temperatur auf d. Stoffwechsel d. Warmblüter. 101

mässig bei den Versuchen bleibt, so liessen wir aus dem calibrirten Maassrohr A so viel Chlorcalciumlösung durch Oeffnen des Hahnes von A in das Druckrohr B tropfenweise ausfliessen, als Sauerstoff verbraucht worden war. Hierbei leitet der Stand des Manometers M mit unfehlbarer Sicherheit. Diese Anordnung gestattet den Gang des Sauerstoffverbrauchs in beliebig kleinen Zeiträumen ablesen zu können. Es ist nothwendig zu bemerken, dass unsere Anordnungen so getroffen waren, dass durch Ausfliessen von Chlorcalciumlösung aus A nicht etwa gleichzeitig Luftblasen durch das Druckrohr B hinüber in Ballon $\mathrm{C}$ getrieben wurden.

Wir bedienten uns bei dieser Untersuchung oft der Methode, dass das Thier an einem Tage zweien Versuchen unterworfen wurde, die zusammen etwa 6-8 Stunden dauerten und während welcher dem Thiere kein Futter verabreicht wurde. Zwischen zwei Versuchen war immer ein Intervall von einigen Stunden, in dem das Thier auch nichts zu fressen erhielt und nicht im Apparat war. Derjenige Versuch, in dem ein energischerer Stoffwechsel nachgewiesen werden sollte, war also immer der zweite am Tage. Wir haben das Thier für den ersten Versuch gewöhnlich, nachdem es ein nahrhaftes Futter zu sich genommen hatte, benutzt, einmal auch, nachdem es seit 12 Stunden keine Nahrung mehr bekommen hatte. Auf diese Weise wurde erreicht, dass der Einfluss, den die so sehr. verschiedene Nahrung der Pflanzenfresser von einem Tag zu dem anderen bedingt, beseitigt war, ebenso wie die anderen periodischen Schwankungen im Gesundheitszustand von einem Tag zum anderen. Nachdem wir eine Reihe von Serien auf diese Weise angestellt hatten, waren noch Versuche nach der Methode nothwendig, welche von Versuch XI bis XX befolgt worden ist.

Was nun die Temperaturen betrifft, deren wir uns bedienten, so verglichen wir immer nur den Stoffwechsel bei der zufällig vorhandenen Zimmertemperatur mit den, welcher sich ergab, wenn das Wasser des Cylinders $\mathrm{P}$ und $\mathrm{W}$ mit Eisstücken gefüllt war. In diesem Falle liessen wir die Temperatur in dem Athemraume des Thiers nicht sehr stark herabgehen, was durch die Menge des schmelzenden Eises leicht regulirt werden kann. Das Thermometer $\mathrm{N}$ zeigte dann $6-8^{\circ} \mathrm{C}$. und wir waren bestrebt auf das sorgfältigste während des ganzen Versuchs diese Temperatur constant zu erhalten. Bei dem Kälteversuch standen auch die Cylinder $Y_{1} Y_{2}$ in mit Eis gefüllten Blecheimern. 
Ehe der Versuch anfangen sollte, wurde der Apparat in Gang gesetzt, während natürlich die Klappe in Fussboden des Thierrecipienten noch nicht ganz geschlossen war, und die Ventilation so lange fortgesetzt, bis das Thermometer einen constanten Stand angenommen hatte, den wir bei den Versuchen einzuhalten wünschten. Darauf wurde das Thier schnell eingeführt, auf Watte gesetzt, die Klappe hermetisch geschlossen und der Kasten Q mit Wasser gefüllt. Stets haben wir dafür gesorgt, das Thier nicht zu starker Kälte auszusetzen, sodass es am Ende des Versuchs in Recto noch annähernd dieselbe Temperatur zeigte, die es vor dem Versuch gehabt hatte. Wir haben zu allen Versuchen Meerschweinchen benutzt, welche sich vermöge der ausserordentlichen Energie ihrer Wärmeregulation für diese Versuche ganz vorzüglich bewährt haben.

Jeder Versuch dauerte gewöhnlich so lange, bis das Thier den Sauerstoff aus der Glaskugel C vollkommen verbraucht hatte. Die Art der Anordnung des Apparats ist aber eine solche, dass man den Versuch fast beliebig lange Zeit fortsetzen könnte. Zu dem Ende müssten nur natürlich neue mit Kalilauge gefüllte Ventile eingeschaltet und in Betreff des Wiederersatzes des aus $C$ verbrauchten Sauerstoffs in einer Weise verfahren werden, analog derjenigen, die Regna ult inne gehalten hat. Nichts destoweniger ist eine Dauer des Versuchs von 3-5 Stunden vollkommen ausreichend zur Gewinnung sicherer Werthe. Um den Einwand zu beseitigen, der gemacht worden ist, dass die Wärmeregulation nur in den ersten Augenblicken nach stattgehabter Temperaturschwankung bemerkbar werde, liessen wir die Thiere, wenn sie einer niedrigen Temperatur im Athemraume $O$ ausgesetzt werden sollten, schon vorher mehrere Stunden im Eiskasten sitzen.

Das Totalvolum des Athemraumes minus Leitung $\mathrm{H}$ und Luftvolum in $G$ ist durch Füllung aller Theile mit Wasser ermittelt und während des Versucht gleich 4293 bis 4443 Cc. gefunden worden. Dieser Werth schwankt natürlich nach der Tiefe, bis zu welcher die Aspiratoren in Quecksilber tauchen und nach dem Volum des Thieres, wobei Gramm $=$ Cc. gesetzt ist.

Der Apparat ist bei verschiedener Temperatur Tage lang unter Druck gestellt worden, zugleich um zu constatiren, dass die von uns in Manometer M zum Abschluss benutzte Chlorealciumlösung keine bemerkbare Menge von Gas durch Diffusion durchlässt und ausserdem, um ganz sicher zu sein, dass die Gummiverbindung an der 
Einfluss d. umgebenden Temperatur auf d. Stoffwechsel d. Warmolüter. 103 eirgeschalteten Vorrichtung $\mathrm{G}$ dicht sei. Hier stiessen die zu verbindenden Röhren unmittelbar aneinander úd das dickwandige in heissem Fett gesottene Gummirohr bet also nur ein Minimum von Oberfläche für Diffussion, die bei den nur höchstens 5-6 Stunden dauernden Versuchen vernachlässigt werden darf. Es wäre übrigens leicht und doch wünchenswerth, auch diese Stelle noch zu eliminiren, was in Zukunft geschehen soll.

\section{B. Gasanalyse.}

Die Gasanalyse wurde nach folgender von Pflüger modificirten Bunsen'schen Methode ausgeführt. Nachdem die aus dem Athemraume mit Hülfe der Kugel $R$ entnommene Luftprobe in der Bunsen'schen Quecksilberwanne in ein weites geschnabeltes Absorptionsrohr übergefüllt worden war, wodurch wir eine Luftmasse von $120 \mathrm{Cc}$. erhielten, wurde ein kleinerer Theil dieses Gases in ein langes Absorptionsrohr gebracht, um in demselben die Kohlensäure nach bekannten Regeln zu bestimmen. In das weite Absorptionsrohr, welches den Rest der aus $R$ erhaltenen Luft enthielt, wurde nachher eine Kalikugel eingefübrt und die Nacht über zur Absorption hingestellt. Auf diese Weise wurde die Kohlensäure dieses nicht gemessenen Gasgemisches vollkommen absorbirt, und das letztere darauf in ein $1 \mathrm{M}$. langes und $15 \mathrm{Mm}$. weites Eudiometer übergefüllt. Nachdem Volum, Druck und Temperatur abgelesen, wurde nach Zusatz von elektrolytischem Knallgas die Explosion bewirkt. Wir haben uns jedesmal an Proben des Knallgases durch einen besonderen Versuch überzeugt, dass es keinen überschüssigen Wasserstoff oder Sauerstoff enthielt. Nach stattgehabter Contraktion des Gases (falls dieselbe vorhanden war), wurde abermals Volum, Druck und 'Temperatur abgelesen, und die neugebildete Kohlensäure mit einigen Cubikcentimetern sehr verdünnter Kalilauge (1,030 sp. G.) absorbirt. Ein Volum feuchter atmosphärischer Luft im Absorptionsrohr gemessen und reducirt, gibt nach $P$ flüg e r denselben Werth, wenn nachher diese Kalilauge eingespritzt und das Gas wieder als ganz feucht berechnet wird. Die Stärke der Kalilauge war von uns durch Titriren festgestellt worden. Nachdem jetzt abermals die Contraction des Gases, die in Folge der Absorption der Kohlensäure eingetreten war, festgestellt, wurde die rückständige atmosphärische Luft nach Bunsen's Methode mit elektrolytischem Wasserstoff verpufft. Der 
nach Buns en vorschriftsmässig bereitete electrolytische Wasserstoff selbst war jedesmal von uns durch Verpuffen mit Knallgas auf $A b$ wesenheit atmosphärischer Luft geprüft worden. Auf diese Weise dauert eine Analyse zwar immer noch mehrere Tage, lässt sich aber doch viel schneller ausführen als bei Anwendung der von $\mathrm{B}$ u n s e n immerbenutzten Kalikugeln. Wenn man Kalikugeln braucht, beansprucht die Ausführung einer solchen Gasanalyse, bei welcher nach einander Kohlensäure, Sumpfgas, nochmals Kohlensäure, Wasserstoff, Saiuerstoff und Stickstoff quantitativ bestimmt werden müssen, wegen der nothwendigen Trocknung der Gase nach der Absorption der Kohlensäure immer eine Reihe von Tagen.

Wàs endlich die von uns befolgte Methode zur Bestimmung der während des Versuches von den Thieren producirten Kohlensäure betrifft, welche von der Kalilauge der Ventile absorbirt wurde, so ist diese Kalilauge in der Quecksilberpumpe mit Phosphorsäure ausgepumpt worden. Da in dem Bonner Laboratorium die Vorrichtungen vorhanden sind, um im Laufe von 2 Tagen 10 bis zu 20 Kohlensäurebestimmungen zu machen, die an Genauigkeit jeder nach anderer Methode gewonnenen überlegen sind, und da die Wägungsund Titrirmethoden, wenn sie wirklich genau ausgeführt werden, so lange es sich um starke kohlensäurehaltige Kalilauge handelt, auch nicht schneller zum Ziele führen, so dürfte diese Methode gerechtfertigt sein.

\section{Untersuchung.}

Wenn es feststände, dass bei dem Athmen der Thiere im abgeschlossenen Raum der Stickstoff keine in Betracht kommende Veränderung seiner Menge erlitte, wenn ferner alle von dem Thier gebildete Kohlensäure sofort absorbirt würde, wenn endlich keine Ex - . halation von Wasserstoff und Kohlenwasserstoff stattfände, Jruck und Temperatur unverändert blieben, würde man das durch die Respiration des Thieres verschwundene Gasvolum unmittelbar als Sauerstoff berechnen dürfen. Selbst angenommen, dass die von Regnault fast allgemein gefundene Stickstoffexhalation auf einem Beobachtungsfehler beruht, was in hohem Maasse wahrscheinlich ist, so würde doch durch Nichtberïcksichtigung dieser Fehlerquelle unser Resultat nicht geschädigt werden. Es handelt sich bei unseren Bestimmungen um die Grösse des Sauerstoffverbrauchs und 
Einfluss d. umgebenden Temperatur auf d. Stoffwechsel d. Warmblüter. 105

der Kohlensäurebildung. Nun ergibt sich aus den Analysen Regnaults, dass auf $1000 \mathrm{Ccm}$. Sauerstoff im Mittel ungefähr $10 \mathrm{Ccm}$. Stickstoff exhalirt werden. Das sind wenigstens die Werthe, die sich aus seinen an Pflanzenfressern, nämlich Kaninchen, angestellten Versuchen ergeben, und für uns zunächst massgebend sein müssen, weil wir mit Meerschweinchen experimentirt haben. Daraus folgt, dass man bei Nichtberücksichtigung der hypothetischen Stickstoffexhalation einen Fehler begeht, der den verbrauchten Sauerstoff um 1/10otel zu klein erscheinen lässt. Obwohl nun diese Stickstoffexhalation überhaupt in hohem Grade zweifelhaft ist, hielten wir, da wir nicht an Kaninchen, sondern an Meerschweinchen experimentirten, über welche keine genaueren Versuche vorliegen, es für nothwendig uns selbst zu überzeugen, $o b$ auf Grund sorgfältig ausgeführter Analysen der am Ende des Versuchs im Apparat enthaltenen Athemluft eine Veränderung im Stickstoffgehalt angenommen werden dürfe. Wir werden die betreffenden Analysen mittheilen, aus denen hervorgeht, dass innerhalb einer Reihe von 3 bis 6 Stunden eine Aenderung in dem procentischen Stickstoffgehalt des Athemraumes nicht angenommen werden kann. Auf Grund dieser Versuche sind wir berechtigt, den Fehler, der durch Veränderung des Stickstoffgehaltes entstehen kann, gleich Null zu setzen.

Was nun die Exhalation von Kohlenwasserstoff und Wasserstoff betrifft, so beobachteten wir dieselbe regelmässig bei allen nicht hungernden Thieren. Die Correctheit verlangt also die Kenntniss des am Ende des Versuchs im Apparat enthaltenen Sumpfgases, des Wasserstoffs und der Kohlensäure. Da aber bei diesen und anderen physiologischen Forschungen viele Versuche an ein und demselben Thier und an ein und demselben und vielen auf einander folgenden Tagen angestellt werden müssen, und $\mathrm{da}$ diess unmöglich wäre, wenn jedesmal die vielen zeitraubenden Analysen der Athemluft gemacht werden müssten, verfährt man zweckmässig, wie hier geschehen: 1. Man bestimmt in vielen Versuchen den Sauerstoffverbrauch genau, soweit er sich durch Verkleinerung des totalen Gasquantums za erkennen gibt. 2. Man bestimmt an denselben Thieren, mit denen man arbeiten will, unter denselben Bedingungen, wie viel brennbares Gas sie pro Kilo und Stunde exhaliren. Dies beträgt für Meerschweinchen nach unseren Analysen im Mittel $=24,6 \mathrm{Cc}$. $\left(0^{\circ}\right.$ C. und $\left.0^{\mathrm{m}}, 77\right)=21,1$ Cc. Sumpfgas $+3,5$ Cc. Wasserstoff. 3. Meerschweinchen exhaliren nüchtern nur Spuren oder gar kein 
brennbares Gas. 4. Unser Apparat enthält am Ende eines 3-5 Stunden dauernden Versuchs immer nahezu $0,5 \%$ Kohlensäure $=$ $15 \mathrm{Cc}$. $\left(0^{0}\right.$ und $\left.0^{\mathrm{m}}, 76 \mathrm{Hg}\right)$. Diess hat seinen Grund darin, dass in fast jedem Versuch dasselbe Sauerstoffvolum verbraucht wird, weshalb Versuchsdauer und Stoffwechsel ungefähr umgekehrt proportional sind. Aus diesen Daten berechnet man leicht, wie viel der Sauerstoff pro Kilo und Stunde im Mittel zu klein erscheint. 5. Die Kohlensäure im Athemraume am Ende des Versuchs ist zu der in der Kalilauge enthaltenen $\mathrm{zu}$ addiren.

Versuch 1 und 2 sind im Protokoll mit allen Berechnungen und Analysen mitgetheilt. Versuch 3-20 enthalten im Protokoll nur die rohen Werthe: Sauerstoff ohne Correctur wegen Sumpfgas, Wasserstoff und Kohlensäurérest im Athemraum; Kohlensäure ohne Correctur wegen dieses Restes.

In Generaltabelle II sind zunächst alle Werthe von Versuch 1-20 noch ohne Correctur angegeben; hieraus sind die rohen Mittel gezogen.

Unter der Tabelle II finden sich dann die corrigirten Mittelwerthe, die wwahren Mittel“, wobei alle Cautelen beachtet sind. Man wird bemeiken, dass die Correctur das Resultat in irrelevanter Weise ändert. Es muss aber natürlich bei jeder naturwissenschaftlichen Untersuchung die absolute Grösse des Beobachtungsfehlers bekannt sein.

Für diejenigen Lèser, welche die Details der nunmehr mitzutheilendēn 20 Versuche nicht zu lesen wünschen, sei hier die Bemerkung gemacht, dass an dem Ende der Abhandlung zwei Generaltabellen zusammengestellt sind. In der zweiten Generaltabelle befinden sich die wichtigsten Werthe, um die es sich handelt, in übersichtlicher Anordnung.

\section{Versuch I.}

Meerschweinchen Nr. I von $451 \mathrm{Gr}$.

Daner des Versuchs: 3 Stunden 14 Minuten.

Rectumtemperatur vor dem Versuch $38.0^{\circ}$.

$\triangleright$ nach dem Versuch $38.2^{3}$.

Mittlere Temperatur des Laboratoriums $=15.9^{0}$.

Temperatur in der Glocke $=15.5^{\circ}$.

Temperatur des Sauerstoffs im Anfang des Versuches $=15.5^{\circ}$.

Barometerstand am Anfang des Versuchs $0^{\mathrm{m}} .7571 ; \mathrm{t}=14.5$. 
Einfluss d. umgebenden Temperatur auf d. Stoffwechsel d. Warmblïter. 107

Analyse der am Ende des Versuches genommenen Luftprobe des Respirationsapparates:

$$
\begin{array}{llr}
\text { Sauerstoff . . } & 18.36 \% \\
\text { Kohlensäure } & \cdot & 0.44 \% \\
\text { Methan . . } & 0.82 \% \\
\text { Wasserstoff . . . } & 0.1 \% \\
\text { Stickstoff . . } & 80.28 \%
\end{array}
$$

Inhalt des Apparates, in welchem das Thier athmet, anf $0^{\circ}$ und $0^{\mathrm{m}} .76$ reducirt $=4160.4 \mathrm{Cc}$.

Das Stickstoffvolum der im Apparat beim Beginn des Versuches enthaltenen atmosphärischen Luft auf $0^{\circ} \mathrm{C}$. und $0.76 \mathrm{M}$. Druck reducirt = 3288.4 Cc.

Stickstoffverunreinigung des während des Versuchs in den Thierbehälter einströmenden Sauerstoffs und Berücksichtigung, dass G and H vor Versuch atmosphärische Luft, am Ende des Versuchs Sauerstoff entbalten, ergibt eine Stickstoffzunahme im Apparat auf $0^{\circ}$ und $0.76 \mathrm{M}$. Druck reducirt $=56.1 \mathrm{Cc}$.

In Folge dieser Verunreinigung musste der Thierbehälter, der beim Beginn des Versuchs 79.04\% N. (atmosphärische Luft) enthielt, am Ende des Versuchs an Stickstoff enthalten $=80.39 \%$.

Der durch obige Analyse gefundene Stickstoffgehalt $(80.28 \%)$ ist für die Luftmassen im Apparate nicht richtig, welche vom Versuchsthier durch die mit Kalilauge gefüllten Ventile getrennt sind, dieser entkohlensäuerte Raum (Aspiratoren + Ventile) $(1200$ Cc.) enthält $0.35 \%$ N. mehr. 'Der mittlere Procentgehalt des Apparates an Stickstoff ist demnach $=80.37 \%$.

Sauerstoffaufnabme im ganzen Versuch 1622.6 Cc.

Sauerstoffverbrauch pro Stunde 501.8 Cc.

Sauerstoffverbrauch pro 1 Kilo Thiergewicht in 1 Stunde $=1112.6 \mathrm{Cc}$.

Kohlensäureabgabe im ganzen Versuch 1566.19 Cc.

Kohlensäureabgabe pro Stunde $484.3 \mathrm{Cc}$.

Kohlensä!reabgabe pro 1 Kilo Thiergewicht in einer Stunde Zeit $=$ $1073.8 \mathrm{Cc}$.

Verhältniss des in der Kohlensäure enthaltenen Sauerstoffs zum verbrauchten Sauerstoff $=0.96$.

Bemerkung: In diesem Versuche beträgt die Abweichung des berechneten vom beobachteten Procentgehalt des Stickstoffs noch nicht $1 / 10 \%$, sondern nur $0,02 \%$.

Das Thier hat unmittelbar vor dem Versuch gefressen, Wasserstoff und Sumpfgas exhalirt.

\section{Versuch II.}

Meerschweinchen No. 1 von $4.49 \mathrm{Gr}$.

Dauer des Versuchs 2 Stunden 25 Minuten.

Rectumtemperatur vor dem Versuch $37^{\circ}$.

$$
\text { } \quad \text { nach dem Versuch 37.1 }{ }^{\circ} \text {. }
$$

Mittlere Temperatur des Laboratoriums $=16.8^{\circ}$. 

$=6.0^{\circ}$.

Temperatur der Glocke (an Thermometer $\varrho$ abgelesen, siehe die Tafel)

Temperatur des Sauerstoffs am Anfang des Versuchs $=17.6^{\circ}$.

Barometerstand am Anfang des Versuchs $0 \mathrm{~m} .7566 ; \mathrm{t}=16.7^{\circ}$.

$$
\text { am Fnde } * \quad 0^{\mathrm{m}} .7566 ; \mathrm{t}=16.7^{\circ} \text {. }
$$

Analyse der am Ende des Versuches aus dem Respirationsapparat genommenen Luftprobe:

$$
\begin{aligned}
& \text { Sauerstoff . . . . . . . . } 19.37 \% \text {. } \\
& \text { Kohlensäure . . . . . . . . . } 0.53 \% \text {. } \\
& \text { Wasserstoff und Methan fehlen . . - } \\
& \text { Stickstoff . . . . . . . . . } 80.1 \% \text {. } \\
& 100.00 \% \text {. }
\end{aligned}
$$

Luft des ganzen Apparates, in welchem das Thier athmet, auf $0^{\circ}$ und $0,7.6 \mathrm{M}$. reducirt $=4238.1 \mathrm{Cc}$.

Das Stickstoffvolum der im Apparat beim Beginn des Versuchs enthaltenen atmosphärischen Lnft auf $0^{\circ}$ und 0.76 M. Druck reducirt = 3349.81 Cc.

Stickstoffverunreinigung des während des Versuchs in den Apparat einströmenden Sauerstoffs und Berücksichtigung, dass $G$ und $H$ am Anfang des Versuchs atmosphärische Luft, am Ende Sauerstoff enthalten, ergibt eine Stickstoffzunahme im Apparat, auf $0^{\circ} \mathrm{C}$. u. $0.76 \mathrm{M}$. Druck reducirt $=42.9 \mathrm{Cc}$.

In Folge dieser Verunreinigung musste der Thierbehälter, der beim Beginn des Versuchs 79.04\% Stickstoff (atmosphärische Luft) enthielt, am Ende des Versuchs an Stickstoff enthalten $=80.05 \%$.

Der durch obige Analyse gefundene Stickstoffgehalt $(80.1 \%)$ ist für die Luft des Apparates nicht richtig, welche vom Versuchsthier durch die mit Kalilauge gefüllten Ventile getrennt ist, dieser entkohlensäuerte Raum = Aspiratoren + Kaliventile $(1200 \mathrm{Cc}$.) enthält $0.42 \%$ N. mehr. Der mittlere Procentgehalt des Apparates ist demnach $=80.21$.

Sauerstoffaufnahme im ganzen Versuch $1570.65 \mathrm{Cc}$.

Sauerstoffverbrauch pro Stunde $649.92 \mathrm{Cc}$.

Sauerstoffverbrauch pro Kilo Thiergewicht und eine Stunde $=1447.4 \mathrm{Cc}$.

Koblensäureabgabe im ganzen Versuch 1382.25 Cc.

Koblensäureabgabe pro Stunde $571.9 \mathrm{Cc}$.

Kohlensäureabgabe pro Kilo Thiergewicht and Stunde $1273.7 \mathrm{Cc}$.

Verbältniss des in der Kohlensäure enthaltenen Sauerstoffs zum verbrauchten Sauerstoff 0.88 .

Bemerkung: Auch hier ist der berechnete and gefundene Stickstoff nur um $0.16 \%$ verschieden. Also ist es bewiesen, dass der Stickstoff sich in keiner bei unsern Versuchen in Betracht kommenden Weise ändert.

Die Versuche I u. II sind an demselben Tage und an demselben Thier angestellt. Vor dem am Nachmittag vorgenommenen Kälte-Versuch befand sich das Thier zwei Stunden im Eiskasten. 
Einfluss d. umgebenden Temperatur auf d. Stoffwechsel d. Warmblüter. 109

\section{Versuch III.}

Meerschweinchen No. I von $415 \mathrm{Gr}$.

Dauer des Versuchs 3 Stunden 35 Minuten.

Rectumtemperatur vor Versuch $37.5^{\circ}$.

Mittlere "Temperatur des Laboratoriums $16.8^{\circ}$.

Temperatur in der Glocke (Thermometer $\varrho$ siehe Tafel) $=16.4^{0}$.

Temperatur des Sauerstoffs im Anfang des Versuchs $=16.5^{\circ}$.

Barometer am Anfang des Versuch $=0 \mathrm{~m} .7564 \mathrm{M} ; \mathrm{t}=15.5^{\circ}$.

$" \quad$ am Ende des Versuchs $=0 \mathrm{~m} .7564 ; \mathrm{t}=15.5^{\circ}$.

Sauerstoffaufnahme im ganzen Versuch $1561.7 \mathrm{Cc}$.

Sauerstoffverbrauch pro Stunde 436.229 Ce.

Sauerstofferbrauch pro Kilo Thiergewicht und 1 Stunde im Cc. $=$ 1051.15. Cc.

Kohlensäureabgabe im ganzen Versuch 1191.24 Cc.

Kohlensäureabgabe pro Stunde $=332.79 \mathrm{Cc}$.

Kohlensäureabgabe pro Kilo Thiergewicht und Stunde $=801.90 \mathrm{Cc}$.

Verbältniss des in der Kohlensäure enthaltenęn Sauerstoffs zum verbranchten Sauerstoff $=0.76$

Das Thier hat seit dem Tage vorher (Abends) nichts gefressen, ist nüchtern.

Versuch IV.

Meerschweinchen (No. I) von $408 \mathrm{Gr}$.

Dauer des Versuchs 2 Stunden 21 Minuten.

Rectumtemperatur vor dem Versuch $37.6^{\circ}$.

" nach dem Versuch $38.0^{\circ}$.

Mittlere Temperatur des Laboratoriums $16.5^{\circ}$.

Temperatur in der Glocke $5.5^{\circ}$.

Temperatur des Sauerstoffs im Anfang des Versuchs 17.2

Barometerstand am Anfang des Versuchs 0,7568; $t=16.5^{\circ}$.

,

am Ende des Versuchs 0.7568; $t=16.5^{\circ}$.

Sa.uerstoffaufnahme im ganzen Versuch 1557.5 Cc.

Sauerstoffverbrauch pro Stunde 662.77 Cc.

Sauerstoffverbrauch pro Kilo Thiergewicht und eine Stunde Zeit $=$ 1624.43 Cc.

Kohlensäureabgabe im ganzen Versuch 1183.64 Cc.

Kohlensäureabgabe pro Stunde $503.68 \mathrm{Cc}$.

Kohlensäureabgabe pro Kilo Thiergewicht und eine Stunde Zeit = 1234.31 Cc.

Verhältniss des in der Kohlensäure enthaltenen Sauerstoffs zum verbrauchten Sauerstoff $=\mathbf{0 . 7 6}$.

Bemerkungen. Das Meerschweinchen ist 2 Stunden ror dem Ver- 
such im Eiskasten gewesen. Der Versuch ist am Nachmittag angestellt, nachdem am Vormittag Versuch I angestellt war.

Das Thier hat seit dem Abend des vorhergehenden Tages nichts gefressen. Versuch III und IV sind an ein und demselben Tage und an ein und demselben Thier angestellt.

\section{Versuch V.}

Meerschweinchen No. II von $382 \mathrm{Gr}$.

Dauer des Versuchs: 4 Stunden 12 Minuten.

Rectumtemperatur vor dem Versuch $37.1^{\circ}$ :

$$
\text { D nach dem Versuch } 37.9^{\circ} \text {. }
$$

Mittlere Temperatur des Laboratoriums $18.8^{\circ}$.

Temperatur in der Glocke (am Thermometer $\varrho$ abgeleaen siehe Tafel) $=18.7^{\circ}$.

Temperatur des Sauerstoffs im Anfang des Versuchs $=18.0^{\circ}$.

Barometerstand am Anfang des Versuchs 0m.7426; $t=17.6^{\circ}$.

$\triangleright \quad$ am Ende des Versuchs $0 \mathrm{~m} .7420 ; t=16.8$.

Sauerstoffaufnahme im ganzen Versuch 1521.6 Cc.

Sauerstoffverbrauch pro Stunde 362.29 Cc.

Sauerstoffverbrauch pro Kilo Thiergewicht und eine Stunde Zeit = 948.17 Ce.

Kohlensäureabgabe in dem ersten Zeitabschnitt des Versuchs $=717.61 \mathrm{Cc}$. $\gg$ in dem zweiten Zeitabschnitt des Versuchs $=$ 681.546 Cc.

Kohlensäureabgabe pro Stunde in dem ersten Zeitabschnitt des Versuchs $=358.80 \mathrm{Ce}$.

Kohlensäureabgabe pro Stunde in dem zweiten Zeitabschnitt des Versuchs $=272.62 \mathrm{Cc}$.

Kohlensäureabgabe pro Kilo Thiergewicht und 1 Stunde Zeit $=872.06 \mathrm{Cc}$.

Kohlensäureabgabe pro Kilo Thiergewicht und 1 Stunde Zeit in dem ersten Zeitabschnitt des Versuchs $=939.27 \mathrm{Cc}$.

Kohlensäureabgabe pro Kilo Thiergewicht und 1 Stunde Zeit in dem zweiten Zeitabschnitt des Versuchs $=713.61 \mathrm{Cc}$.

Verhältniss des in der während des ganzen Versuchs abgegebenen Kohlensäure enthaltenen Sauerstoffs zu dem während des ganzen Versuchs verbrauchten Sauerstoff $=0.92$.

\section{Versuch VI.}

Meerschweinchen No. II von $379 \mathrm{Gr}$.

Dauer des Versuchs 2 Stunden 53 Minuten.

Rectumtemperatur am Anfang des Versuchs $37.4^{\circ}$.

، am Ende des Versuchs 38.2.

Mittlere Temperatur des Laboratoriums $18.2^{\circ}$.

Temperatur in der Glocke (am Thermometer $\varrho$ abgelesen siehe Tafel) $=10^{\circ}$. 
Einfluss d umgebenden Temperatur auf d. Stoffwechsel d. Warmblüter. 111

Temperatur des Sauerstoffs am Anfang des Versuchs $=19.2^{\circ}$.

Barometerstand am Anfang des Versuchs 0m.7416; $\mathrm{t}=18.7^{\circ}$.

$\gg \quad$ am Ende des Versuchs 0m.7416; $t=18.7^{\circ}$.

Sauerstoffverbrauch im ganzen Versuch $=1510.7 \mathrm{Cc}$.

$\gg \quad$ pro Stunde $=524.55 \mathrm{Cc}$. .

D proKilo Thiergewicht und 1 Stunde Zeit $=1384.77 \mathrm{Cc}$.

Kohlensäureabgabe in dem ersten Abschnitt des Versuchs $=629.33 \mathrm{Cc}$. in dem zweiten Abschnitt des Versuchs $=653.31 \mathrm{Cc}$.

Kohlensäureabgabe pro Stunde in dem ersten Abschnitt des Versuchs $=473.18 \mathrm{Cc}$.

Kohlensäureabgabe pro Stunde in dem zweiten Abschnitt des Versuchs' $=424.78 \mathrm{Co}$.

Kohlensäureabgabe pro Kilo Thiergewicht und Stunde $=1178.34 \mathrm{Cc}$.

Abschnitt des Versuchs $=1248.49$ C.

Kohlensäureabgabe pro Kilo Thiergewicht und Stunde in dem zweiten Ábschnitt des Versuchs $=1120.08 \mathrm{Cc}$.

Volumverhältniss der während des ganzen Versuchs abgegebenen Kohlensäure zu dem während des ganzen Versuchs verbrauchten Sauerstoff $=0.85$.

Bemerkung: Versuch 5 und 6 sind an ein und demselben Tage mit ein und demselben Thiere angestellt. Das Thier befand sich vor Versuch 6 mehrere Stunden im Eiskasten.

\section{Versuch VII.}

Meerschweinchen No. II von $400 \mathrm{Gr}$.

Dauer des Versuchs 2h für Kohlensäure, $3 \mathrm{~h} 23 \mathrm{~m}$ für Sauerstoff.

Rectumtemperatur am Anfang des Versuchs $=38^{0} .5$. am Ende des Versuchs $=38^{\circ}$.

Mittlere Temperatur des Laboratoriums $18.4^{\circ}$.

Temperatur der Glocke (an Thermometer $\varrho$ abgelesen siehe Tafel) $17.5^{\circ}$.

Temperatur des Sauerstoffs im Anfang des Versuchs $=18.4^{\circ}$.

Barometerstand am Anfang des Versuchs $0 \mathrm{~m} .7525 ; \mathrm{t}=18.2^{\circ}$.

$\gg \quad$ am Ende des Versuchs $0 \mathrm{~m} .7525 ; \mathrm{t}=18.2^{\circ}$.

Sauerstoffiverbrauch im ganzen Versuch $=1544.8$ Cc.

Sauerstoffverbrauch pro Stunde $=455.55 \mathrm{Cc}$.

Sauerstoffverbrauch pro Kilo Thiergewicht und 1 Stunde $=1138.87 \mathrm{Cc}$.

Kohlensäureabgabe in den 2 Stunden des Versuchs $=759.60 \mathrm{Cc}$.

Kohlensäureabgabe pro Stunde berechnet $=379.8 \mathrm{Cc}$.

Kohlensäureabgabe pro Kilo Thiergewicht und 1 Stunde berechnet $=949.5 \mathrm{Ce}$.

Die Kohlensäure für die letzte Hälfte des Versuchs konnte nicht bestimmt werden durch einen Unfall (Zerspringen eines Ventiles).

Volumverhältniss der exhalirten Kohlensäure zu dem verbranchten Sauerstoff $=0.83$ 


\section{Versueh VIII.}

Meerschweinchen No. II von $395 \mathrm{Gr}$.

Dauer des Versuchs 3 Stunden 29 Minuten.

Rectumtemperatur vor dem Versuch $38.5^{\circ}$.

$$
\text { " nach dem Versuch } 38^{\circ} \text {. }
$$

Mittlere Temperafur des Laboratoriums $18.7^{\circ}$.

Temperatur in der Glocke (am Thermometer $\varrho$ abgelesen siehe Tafel) 7.5 .

Temperatur des Sauerstoffs im Anfang des Versuchs 19.3 .

Barometerstand am Anfang des Versuchs $0^{\mathrm{m}} .756$; t $18.5^{\circ}$.

$\gg \quad$ am Ende des Versuchs $0 \mathrm{~m} .756 ; t=18.4^{\circ}$.

Sauerstoffaufnahme im ganzen Versuch $=1542.7 \mathrm{Cc}$.

Sauerstoffverbrauch pro Stunde $=440.77 \mathrm{Cc}$.

Sauerstoffverbrauch pro Kilo Thiergewicht und 1 Stunde Zeit $=1158.73$ Cc.

Kohlensäureabgabe im ganzen Versuch $=1399.44$ Cc.

Kohlensäureabgabe pro Stunde $=399.84 \mathrm{Cc}$.

Kohlensäureabgabe pro Kilo Thiergewicht und 1 Stunde Zeit $=1012.25$ Cc.

Verhältniss des in der während des ganzen Versuchs abgegebenen

Kohlensäure enthaltenen Sauerstoffs zu dem während des ganzen Versuchs verbrauchten Sauerstoff $=0.87$.

Bemerkung: Versuch 7 und 8 sind an einem und demselben Tage an ein und demselben Thier angestellt. Vor Versuch 8 sass das Thier mehrere Stunden im Eiskasten.

\section{Versuch IX:}

Meerschweinchen No. I von $450 \mathrm{Gr}$.

Dauer des Versuchs 3 Stunden.

Rectumtemperatur vor dem Versuch $37.1^{\circ}$. nach dem Versuch $37^{\circ}$.

Mittlere Temperatur des Laboratoriums $16.2^{\circ}$.

Temperatur in der Glocke (am Thermometer $\varrho$ abgelesen, s. Tafel) $16.2^{\circ}$.

Temperatur des Sauerstoffs im Anfange des Versuchs $16^{\circ}$.

Barometerstand am Anfang des Versuchs $0^{\mathrm{m}} .7534, t=15.4^{0}$.

» am Ende des Versuchs $0 \mathrm{~m} .7531 ; \mathrm{t}=16.2^{\circ}$.

Sauerstoffaufnahme im ganzen Versuch $1559.2 \mathrm{Cc}$.

Sauerstoffrerbrauch pro Stunde 519.73 Cc.

Sauerstoffverbrauch pro Kilo Thiergewicht und 1 Stunde Zeit 1154.95 Cc.

Kohlensäureabgabe im ganzen Versuch 1307.1 Cc.

Kohlensäureabgabe pro Stunde $435.7 \mathrm{Cc}$.

Kohlensäureabgabe pro Kilo Thiergewicht und 1 Stunde Zeit 968.22 Cc.

Verhältniss des in der abgegebenen Kohlensäure enthaltenen Sauerstoffs zu dem verbrauchten Sauerstoff 0.84 .

\section{Versuch X.}

Meerschweinchen No. I von $446 \mathrm{Gr}$.

Dauer des Versuchs 2 Stunden 21 Minuten. 
Einfluss d. umgebenden Temperatur auf d. Stoffwechsel d. Warmblüter. 113

Rectumtemperatur vor dem $\nabla$ ersuch $37.6^{\circ}$. nach dem Versuch $38.5^{\circ}$.

Mittlere Temperatur des Laboratoriums $16.3^{\circ}$.

Temperatur in der Glocke (am Thermometer $\boldsymbol{o}$ abgelesen, s. Tafel) $6.17^{\circ}$.

Temperatur des Sauerstoffs am Anfang des Versuchs 16.5 .

Barometerstand am Anfang des Versuchs 0m.753; $\mathrm{t}=16.1^{\circ}$.

D. am Ende des Versuchs $0^{\mathrm{m}} .7526 ; t=158^{\circ}$.

Sauerstoffaufnahme im ganzen Versuch $1555.4 \mathrm{Cc}$.

Sauerstoffaufnahme pro Stunde 661.87 Ce.

Sauerstoffaufnahme pro Kilogr. Thiergewicht und 1 Stunde $1483.86 \mathrm{Cc}$.

Kohlensäureabgabe im ganzen Versuch $1129.08 \mathrm{Cc}$.

Kohlensäureabgabe pro Stunde 480.46 Cc.

Koblensäureabgabe pro Kilogr. Thiergewicht und 1 Stunde 1077.26 Cc.

Verhältniss des in der abgegebenen Kohlensäure enthaltenen Sauerstoffs zum verbrauchten Sauerstoff 0.72 .

Bemerkung: Versuch IX r. $X$ sind an ein und demselben Tage an ein und demselben Thier angestellt. Vor Versuch $\mathrm{X}$ hat das Meerschweinchen mehrere Stunden im Eiskasten gesessen.

\section{Versuch XI.}

Meerschweinchen No. III von $278 \mathrm{Gr}$.

Dauer des Versuchs 5 Stunden 18 Minuten.

Rectumtemperatur vor dem Versuch $37.0^{\circ}$.

D nach dem Versuch 37.50.

Mittlere Temperatur des Laboratoriums $22.0^{\circ}$.

Temperatur der Glocke (am Thermometer $\varrho$ abgelesen, s. Tafel) 22.20

Temperatur des Sauerstoffgases im Anfang 21.6

Barometerstand am Anfang des Versuchs $0 \mathrm{~m} .768 ; \mathrm{t}=19.6^{\circ}$.

a $\quad$ am Ende des Versuchs $0 \mathrm{~m} .768 ; t=19.6^{\circ}$.

Samerstoffaufnahme im ganzen Versuch 1550.6 Cc.

Sauerstoffverbrauch pro Stunde $292.56 \mathrm{Cc}$.

Sauerstoffverbrauch pro Kilo Thiergewicht und 1 Stunde $1052.37 \mathrm{Cc}$.

Kohlensäureabgabe im ganzen Versuch 1277.64 Ce.

Kohlensäureabgabe pro Stunde $241.08 \mathrm{Cc}$.

Kohlensäureabgabe pro 1 Kilo Thiergewicht in einer Stunde Zeit 867.19 Cc.

Verhältnisse des in der Kohlensäure enthaitenen Sauerstoffs zum verbrauchten Sauerstoff 0.82 .

Versuch XII.

Meerschweinchen No. III von $278 \mathrm{Gr}$.

Dauer des Versuchs 3 Stunden 30 Minuten.

Rectumtemperatur am Anfang des Versuchs 36.20.

$\quad$ am Ende des Versuchs $36.3^{\circ}$.

Mittlere Temperatur des Laboratoriums 21.20.

E. Pflüger, Archiv für Physiologie, Ba. XIV. 
Temperatur in der Glocke (am Thermometer $\varrho$ abgelesen, s. Tafel) $6.3^{\circ}$.

Temperatur des Sauerstoffs am Anfang des Versuchs 20.5 .

Barometerstand am Anfang des Versuchs $0 \mathrm{~m} .7648 ; \mathrm{t}=20.4^{\circ}$.

D am Ende des Versuchs $0 \mathrm{~m} .7648 ; \mathrm{t}=20.4^{\circ}$.

Sauerstoffaufnahme im ganzen Versuch 1549.4 Cc.

Sauerstoffverbrauch pro Stunde $442.67 \mathrm{Cc}$.

Sauerstoffverbrauch pro 1 Kilo Thiergewicht in einer Stunde Zeit 1592.33 Cc.

Kohlensäureabgabe im ganzen Versuch 1236.4 Cc.

Kohlensäureabgabe pro Stunde $353.2 \mathrm{Cc}$.

Kohlensäureabgabe pro 1 Kilo Thiergewicht in einer Stunde Zeit $1230 \mathrm{Cc}$.

Verhältniss des in der Kohlensäure enthaltenen Sauerstoffs zum verbrauchten Sauerstoff 0.77 .

Bemerkung. Versuch XI u. XII sind an demselben Thier, aber nicht an demselben, sondern an zwei aufeinander folgenden Tagen angestellt. Versuch XII ist der spätere. Das Thier erhielt immer dasselbe Futter ausser der Versuchszeit, so viel es fressen wollte. In der Glocke bekamen die Thiere nie etwas. Das Meerschweinchen sass mehrere Stunden vor Versuch XII im Eiskasten.

\section{Versuch XIII.}

Meerschweinchen No. III von $224 \mathrm{Gr}$.

Dauer des Versuchs 6 Stunden 8 Minuten.

Rectumtemperatur am Anfang des Versuchs 36.0

$\quad$ am Ende des Versuchs 36.20.

Mittlere Temperatur des Laboratoriums $21.4^{0}$.

Temperatur der Glocke (am Thermometer $\varrho$ abgelesen, s. Tafel) 21.10.

Temperatur des Sauerstoffs im Anfang des Versuchs $20.7^{\circ}$.

Barometerstand am Anfang des Versuchs $0^{\mathrm{m}} .7645 ; \mathrm{t}=20.6^{\circ}$.

$\triangleright \quad$ am Ende des Versuchs $0^{\mathrm{m}} .7624 ; t=21.4^{\circ}$.

Sauerstoffaufnahme im ganzen Versucb 1547.1 Ce.

Sauerstoffverbrauch pro Stunde $252.26 \mathrm{Cc}$.

Sauerstoffverbranch pro Kilo Thiergewicht und Stunde $1144.0 \mathrm{Cc}$.

Kohlensäureabgabe während des ganzen Versuchs $1245.82 \mathrm{Cc}$.

Kohlensäureabgabe in dem ersten Abschnitt des Versuchs 626.92 Cc. \ in dem zweiten Abschnitt des Versuchs $618.9 \mathrm{Cg}$.

Kohlensäureabgabe pro Stunde in cem ersten Abschnitt des Versuchs 219.21 Ce.

Kohlensäureabgabe pro Stunde in dem zweiten Abschnitt des Versuchs 184.74 Cc.

Kohlensäureabgabe pro Kilo Thiergewicht und Stunde in dem ersten Abschnitt des Versuchs 978.6 Cc.

Kohlensäureabgabe pro Kilo Thiergewicht und Stunde in dem zweiten Abschnitt des Versuchs 824.73 Cc.

Verhältniss des in der producirten Kohlensäure enthaltenen Sauerstofts zum verbrauchten Sauerstoff 0.80 . 
Einfluss d. umgebenden Temperatur auf d. Stoffwechsel d. Warmblüter. 115

B emerkung. Versuch XI, XII u. XIII sind also an drei auf einander folgenden Tagen an demselben Meerschweinchen angestellt. Versuch XIII kommt zeitlich nach XII.

Versuch XIV.

Meerschweinchen No. IV von 304 Gr.

Dauer des Versuchs 3 Stunden 6 Minuten.

Rectumtemperatur vor dem Versuch $37.4^{0}$.

D nach dem Versuch $37.0^{\circ}$.

Mittlere Temperatur des Laboratoriums 22.10.

Temperatur der Glocke (am Thermometer $\varrho$ abgelesen, sịehe Tafel) 6.60。

Temperatur des Sauerstoffs im Anfang des Versuchs 22.20.

Barometerstand am Anfang des Versuchs $0 \mathrm{~m} .763 ; \mathrm{t}=21.6^{\circ}$.

$\gg \quad a m$ Ende des Versuchs $0^{\mathrm{m}} .763 ; t=21.6^{0}$.

Sanerstoffaufnahme im ganzen Versuch 1536.2 Cc.

Sauerstoffverbrauch pro Stunde 495.55 Cc.

Sanerstoffverbrauch pro 1 Kilo Thier in einer Stunde Zeit 1630.1 Cc.

Kohlensäureabgabe während des ganzen Versuchs $1470.08 \mathrm{Cc}$.

Kohlensäureabgabe in dem ersten Abschnitt des Versuchs 700.88 Cc.

¿ in dem zweiten Abschnitt des Versuchs 769.20 Cc.

Kohlensäureabgabe pro Stunde in dem ersten Abschnitt des Versuchs 483.36 Cc.

Kohlensäureabgabe pro Stunde in dem zweiten Abschnitt des Versuchs $466.18 \mathrm{Ce}$.

Kohlensäureabgabe pro Kilo Thiergewicht und Stunde in dem ersten Abschnitt des Versuchs $1590 \mathrm{Cc}$.

Kohlensäureabgabe pro Kilo Thiergewicht und Stunde in dem zweiten Abschnitt des Versuchk 1533.48 Cc.

Kohlensäureabgabe pro 1 Kilo Thier und 1 Stunde 1559.93 Cc.

Verhältniss des in der während des ganzen Versuchs abgegebenen Kohlensäure enthaltenen Sauerstoffs zu dem im ganzen Versuch verbrauchten Sauerstoff 0.95 .

Bemerkung. Das Meerschweinchen bat mehrere Stunden vor dem Versuch im Eiskasten gesessen.

\section{Versuch XV.}

Meerschweinchen No. IV, von $292 \mathrm{Gr}$.

Dauer des Versuchs 4 Stunden 45 Minuten.

Rectumtemperatur vor Anfang des Versuchs $37.6^{\circ}$.

D nach Beendigung des Versuchs $37^{\circ}$.

Temperatur des Laboratoriums $20.6^{\circ}$.

Temperatur der Glocke (am Tkermometer $\varrho$ abgelesen, s. Tafel) 21.23.

Temperatur des Sauerstoffs am Anfang des Versuchs $203^{\circ}$.

Barometerstand am Anfang des Versuchs 0m.7568; $\mathrm{t}=19.8^{\circ}$.

" am Ende des Versuchs 0m.7568; $\mathrm{t}=19.8^{\circ}$.

Sauerstoffverbrauch im ganzen Versuch $1537.3 \mathrm{Cc}$. 
Sauerstoffaufnahme pro Stunde 322.64. Cc.

Sanerstoffaufnahme pro Kilo Thiergewicht und 1 Stunde $1108.36 \mathrm{Cc}$.

Kohlensäureabgabe während des ganzen Versuchs $1438.33 \mathrm{Cc}$.

Kohlensänreabgabe in dem ersten Abschnitt des Versuchs 877.17 Cc. » in dem zweiten Abschnitt des Versuchs 561.16 Co.

Koblensäureabgabe pro Stunde in dem ersten Abschnitt des Versuchs 313.27 Cc.

Kohlensäureabgabe pro Stunde in dem zweiten Abschuitt 288 Cc.

Kohlensäureabgabe pro 1 Kilo Thiergewicht und 1 Stunde 1037.00.

Kohlensäureabgabe pro Kilo und Stunde in dem ersten Abschnitt des Versuchs 1072.84 Cc.

Kohlensäureabgabe pro Kilo und Stunde in dem zweiten Abschnitt des Versuchs 986.3 Co.

Verhältniss des in der abgegebenen Kohlensäure enthaltenen Sauerstoffs zum verbrauchten Sauerstoffs 0.93 .

Bemerkung. Versuch XIV u. XV sind an 2 Tagen an ein und demselben Meerschweinchen angestellt. Zwischen Versuch XIV u. XV liegt ein Tag. Versuch XVI.

Meerschweinchen No. IV von $284 \mathrm{Gr}$.

Dauer des Versuchs 3 Stunden 21 Minuten.

Rectumtemperatur vor Anfang des Versuchs $37^{\circ}$. $\gg \quad$ nach Beendigung des Versuchs $37.3^{\circ}$.

Mittlere Temperatur des Laboratoriums $19.4^{\circ}$.

Temperatur der Glocke (am Thermometer $\varrho$ abgelesen, siehe Tafel) 8.40.

Temperatur des Sauerstoffs im Anfange des Versuchs $19.3^{\circ}$.

Barometerstand am Anfange des Versuchs 0m.763; $t=19.5^{\circ}$.

" am Ende des Versuchs 0m.7638; $t=19.8^{\circ}$.

Sauerstoffaufnahme im ganzen Versuch $1551.3 \mathrm{Cc}$.

Sauerstoffaufnahme pro Stunde $483.27 \mathrm{Cc}$.

Sauerstoftaufnahme pro Kilo Thiergewicht und 1 Stunde 1701.65 Cc.

Kohlensäureabgabe während des ganzen Versuchs $1360.30 \mathrm{Cc}$.

Kohlensäureabgabe in dem ersten Abschnitt des Versuchs 610.8 Cc.

» in dem zweiten Abschnitt des Versuchs 749.5 Cc.

Kohlensäureabgabe pro Stunde in dem ersten Abschnitt des Versuchs 381.76 Ce.

Kohlensäureabgabe pro Stunde in dem zweiten Abschnitt des Versuchs 428.28 Cc.

Kohlensäureabgabe pro Kilo und Stunde in dem ersten Abschnitt des Versuchs 1344.19 Cc.

Kohlensäureabgabe pro Kilo und Stunde in dem zweiten Abschnitt des Versuchs 1508.03 Cc.

Kohlensäureabgabe pro 1 Kilo Thier und 1 Stunde $1429.80 \mathrm{Cc}$.

Verhältniss zwischen dem in der abgegebenen Kohlensäure enthaltenen Sauerstoff zum verbrauchten Sauerstoff 0.87 . 
Einfluss d. umgebenden Temperatur auf d. Stoffwechsel d. Warmblüter. 117

Bemerkung. Versuch XIV, XV u, XVI sind an demselben Thier an 3 Tagen angestellt. Zwischen Versuch XIV und XV liegt 1 Tag. Versuch $X V$ und XVI sind an 2 auf einander folgenden Tagen ausgeführt. Das Meerschweinchen sass mehrere Stunden vor Versuch XVI im Eiskasten.

\section{Versuch XVII.}

Meerschweinchen No. IV von $299 \mathrm{Gr}$.

Dauer des Versnchs 2 Stunden 12 Minuten.

Rectumtemperatur vor Anfang des Versuchs $37.5^{\circ}$.

- nach Beendigung des Versuchs 37.10.

Mittlere Temperatur des Laboratoriums $20^{\circ}$.

Temperatur der Glocke (am Thermometer $\varrho$ abgelesen, siehe Tafel) 8.1 ${ }^{\circ}$.

Temperatur des Sauerstofts am Anfang des Versuchs 19.8 .

Barometerstand am Anfang des Versuchs $0 \mathrm{~m} .764 ; \mathrm{t}=19.8^{\circ}$.

» am Ende des Versuchs 0 m.764; $t=19.8^{\circ}$.

Sauerstoffaufnahme im ganzen Versuch 1004.5 Cc.

Sanerstoffaufnahme pro Stunde 456.5 Cc.

\ pro Kilo Thiergewicht und Stunde $1526.7 \mathrm{Cc}$.

Kohlensäureabgabe im ganzen Versuch $894.37 \mathrm{Cc}$.

$\checkmark \quad$ pro Stunde $406.53 \mathrm{Cc}$.

». pro Kilo Thiergewicht und Stunde $1359.63 \mathrm{Cc}$.

Verhältniss des in der abgegebenen Kohlensäure enthaltenen Sauerstoffs zum verbrauchten Sauerstoff 0,88 .

Bemerkung. Versuch XVII ist wie XVI, XV u. XIV an demselben Thier gemacht. Versuch XVI u. XVI wurden an 2 auf einander folgenden Tagen ausgeführt. Das Thier sass mehrere Stunden vor diesem Versuch XVII im Eiskasten.

\section{Versuch XVIII.}

Meerschweinchen No. IV von $293 \mathrm{Gr}$.

Dauer des Versuchs 3 Stunden 22 Minuten.

Rectumtemperatur vor Anfang des Versuchs $37.7^{\circ}$.

D nach Beendigung des Versuchs $37^{\circ}$.

Mittlere Temperatur des Laboratoriums $20.8^{\circ}$.

Temperatur der Glocke (am Thermometer $\varrho$ abgelesen, siehe Tafel) 8.4 .

Temperatur des Sanerstoffs am Anfang des Versuchs 20.70.

Barometerstand am Anfang des Versuchs 0m.764; $\mathrm{t}=20.5^{\circ}$.

D am Ende des Versuchs 0m.7625; $t=20: 6^{\circ}$.

Sauerstoffaufnahme im ganzen Versich 1546.8 Cc.

Sauerstoffaufnahme pro Stunde 460.35 Cc.

Sauerstoffaufnahme pro Kilo Thiergewicht und eine Stunde $1571.16 \mathrm{Cc}$.

Kohlensäureabgabe während des ganzen Versuchs $1500.15 \mathrm{Cc}$.

Kohlensäureabgabe in dem ersten Abschnitt des Versuchs 970.64 Cc.

> in dem zweiten Abschnitt des Versuchs $529.51 \mathrm{Cc}$. 
Kohlensäureabgabe pro Stunde in dem ersten Abschnitt des Versuchs $458.71 \mathrm{Cc}$.

Kohlensäureabgabe pro Stunde in dem zweiten Abschnitt des Versuchs $423.6 \mathrm{Cc}$.

Kohlensäureabgabe pro Kilo Thiergewicht and Stunde in dem ersten Abschnitt des Versuchs 1565.20 Cc.

Koblensäureabgabe pro Kilo Thiergewicht und Stunde in dem zweiten Abschnitt des Versuchs $1445.73 \mathrm{Cc}$.

Kohlensäureabgabe pro 1 Kilo Thier und 1 Stunde $1520.77 \mathrm{Cc}$.

Verbältniss des in der Kohlensäure enthaltenen Sauerstoffs zum verbrauchten Sauerstoff 0.96 .

Bemerkung. Versuch XVIII ist mit XVII, XVI, XV u. XIV an demselben Thier angestellt. Versuch XVIII a. XVII sind an demselben Tage ausgeführt. Das 'Thier hat zwischen Versuch XVII u. XVIII nicht gefressen, sass aber mehrere Stunden vor Versuch XVIII im Eiskasten.

\section{Versuch XIX.}

Meerschweinchen No. IV von $300 \mathrm{Gr}$.

Dauer des Versuchs 4 Stunden 41 Minuten.

Rectumtemperatur am Anfang des Versuchs $38^{\circ}$.

- am Ende des Versuchs $37.8^{\circ}$.

Mittlere Temperatur des Laboratoriums $22.7^{\circ}$.

Temperatur der Glocke (am Thermometer $\varrho$ abgelesen, siebe Tafel) $20.7^{\circ}$.

Temperatur des Sauerstoffs am Anfang des Versuchs $20.4^{\circ}$.

Barometerstand am Anfang des Versuchs 0m.7625; $t=20^{\circ}$.

\ am Ende des Versuchs $0^{\mathrm{m}} .7605 ; t=20^{\circ}$.

Sauerstoffaufnahme im ganzen Versuch $1547.8 \mathrm{Cc}$.

Sacerstoffaufnahme pro Stunde $351.78 \mathrm{Cc}$.

Sauerstoffaufnahme pro Kilo Thiergewicht und eine Stunde 1172.6 Cc.

Kohlensäureabgabe für den ganzen Versuch $781.28 \mathrm{Cc}$.

Kohlensäureabgabe in dem ersten Abschnitt des Versuchs : Bestimmung verunglückt.

Kohlensäureabgabe in dem zweiten Abschnitt des Versuchs $781.28 \mathrm{Ce}$.

Kohlensäureabgabe pro Stunde in dem ersten Abschnitt des Versuchs: Bestimmung verunglückt.

Kohlensäureabgabe pro Stunde in dem zweiten Abschnitt des Versuchs 335.31 Cc.

Kohlensäureabgabe pro Kilo und Stunde in dem ersten Abschnitt des Versuchs: Bestimmung verunglückt.

Koblensäureabgabe pro Kilo und Stunde in dem zweiten Abschnitt des Versuchs 1117.70 Cc.

Verhältniss des in der abgegebenen Kohlensäure enthaltenen Sauerstoffs zam verbrauchten Sanerstoff 0.95 . 
Einfluss d. umgebenden Temperatur auf d. Stoffwechsel d. Warmblüter. 119

Bemerkung. Dieser Versuch ist mit demselben Meerschweinchen wie Versuch XIV, XV, XVI, XVII u. XVIII angestellt. Versuch XVIII u. XIX sind an auf einander folgenden Tagen angestellt.

\section{Versuch XX.}

Meerschweinchen No. IV von $297 \mathrm{Gr}$.

Dauer des Versuchs 6 Stunden 11 Minuten.

Rectumtemperatur am Anfang des Versuchs $37^{\circ}$.

D am Ende des Versuchs $37.8^{\circ}$.

Mittlere Temperatur des Laboratoriums $21.1^{\circ}$.

Temperatur der Glocke (am Thermometer $\varrho$ abgelesen, siehe Tafel) 9.10.

Temperatur des Sauerstoffs am Anfang des Versuchs 21.0 $0^{\circ}$.

Barometerstand am Anfang des Versuchs 0m.7605; $\mathrm{t}=20.0^{\circ}$.

" am Ende des Versuchs 0m.7605; $t=20.0^{\circ}$.

Sauerstoffaufnahme im ganzen Versuch $3087.2 \mathrm{Cc}$.

Sauerstoffaufnahme pro Stunde $499.3 \mathrm{Cc}$.

Sauerstoffanfnahme pro 1 Kilo Thiergewicht und 1 Stunde Zeit $1680.8 \mathrm{Cc}$.

Kohlensäureabgabe im ganzen Versuch $2736.77 \mathrm{Cc}$.

$\gg \quad$ pro 1 Kilo Thiergewicht u. 1 Stunde Zeit 1490.23 Cc.

Kohlensäureabgabe pro 1 Kilo Thiergewicht und 1 Stunde Zeit in dem ersten Zeitabschnitt des Versuchs $1550.37 \mathrm{Cc}$.

Kohlensâureabgabe pro 1 Kilo Thiergewicht und 1 Stunde Zeit im folgeuden Zeitabschnitt des Versuchs $1453.33 \mathrm{Cc}$.

Verhältniss des in der Kohlensäure enthaltenen Sauerstoffs zum verbrauchten Sauerstoff 0.88 .

Bemerkung. Dies ist einer der wichtigsten Versuche, weil das Thier, nachdem è mehrere Stunden im Eiskasten gesessen hatte, länger als 8 Stunden einer Temperatur von $9.1^{\circ} \mathrm{C}$. ausgesetzt gewesen ist und trotzdem einen maximalen Sauerstoffverbrauch und Kohlensäureproduction aufweist. Ausserdem wurde constatirt, dass auch in der späteren Zeit des Versuchs die Kohlensäureproduction immer noch ausserordentlich hoch blieb.

(Der Leser wird gebeten, hier Kenntniss von den beiden Generaltabellen p. 123. 124, am Schluss dieses Aufsatzes, zu nehmen.) 


\section{Resultate.}

I. Meerschweinchen verbrauchen unter normalen Verhältnissen auf 1 Kilo Gewicht und 1 Stunde Zeit bei mittlerer Temperatur = $18.8^{\circ}$ C. -1127.4 Cc. Sauerstoff $=1.612$ Gr. Dieser Werth ist das Mittel aus den in der Generaltabelle als hohe Temperaturen bezeichneten Werthen, da sie der Sommer-Temperatur des Laboratoriums entsprachen.

II. Meerschweinchen erzeugen unter normalen Verhältnissen auf 1 Kilo Gewicht und 1 Stunde bei mittlerer Temperatur $964.15 \mathrm{Cc}$. Kohlensäure $=1.896 \mathrm{Gr}$.

III. Bei Meerschweinchen ist unter normalen Bedingungen das mittlere Verhältniss des in der producirten Kohlensäure enthaltenen Sauerstoffs zum verbrauchten Sauerstoff $=0.86 \mathrm{Gr}$.

IV. Bei Abnahme der Temperatur der Luft verbraucht ein Meerschweinchen für $1^{\circ} \mathrm{C}$. auf 1 Kilo Gewicht und 1 Stunde Zeitmehr:

Sauerstoff . . . . . . . . . . . . . $37.23 \mathrm{Cc}$.

und producirt mehr Kohlensäure . . . . . . $33.66 \mathrm{Cc}$.

Wenn mit abnehmender Temperatur dieser Zuwachs gleichmässig ist, oder zwischen Temperatur und Stoffwechsel umgekehrte Proportionalität stattfindet, würde ein Sinken der Temperatur um $30.2^{\circ} \mathrm{C}$. eine Verdoppelung des Stoffwechsels erzeugen.

V. Das Verhältniss des in der ausgeathmeten Kohlensäure enthaltenen Sauerstoffs zu dem gleichzeitig absorbirten Sauerstoff bleibt, wenn in Folge der Wärmeregulation der Stoffwechsel steigt und sinkt constant $=0.86$. - Diess ergiebt sich aus Versuch XI bis XX (und III u. IV), weil alle Werthe an Thieren in nahe gleicher Verdauungsphase gewonnen sind.

Die Abweichung in der zweiten Decimale ( 0.86 und 0.87 ) ist so winzig, dass erst Versuche mit grösseren Temperaturdifferenzen die mit der Wärmeproduction wachsende Grösse des „respiratorischen Quotienten“ sichern könnten. Wenn man aber erwägt, dass das Volumverhältniss des Kohlensäurezuwachses zum Sauerstoffzuwachs bei wachsender Wärmeproduction nach unseren Versuchen $=0.90$, und dass doch höchst wahrscheinlich die Temperatur regulirende 
Einfluss d. umgebenden 'Temperatur auf d. Stoffwechsel d. Warmblüter. 121

Wärmeproduction durch Innervation der Muskeln bedingt ist, dann erscheint jene spurweise angedeutete Zunahme des respiratorischen Quotienten gar nicht unwahrscheinlich.

VI. Das Verhältniss des in der ausgeathmeten Kohlensäure enthaltenen Sauerstoffs zu dem gleichzeitig absorbirten Sauerstoff ist bei nüchternen Thieren kleiner, aber auch constant bei verschiedener Temperatur. Die Zuwächse der Sauerstoffabsorption sind bei Variation der Temperatur bei nüchternem Zustande sehr wenig kleiner als im nicht nüchternen Zustande. Da nun dieselbe Sauerstoffmenge mehr Wärme (ca. 1/3) erzeugt, wenn Wasserstoff statt Kohlenstoff oxydirt wird, so deutet dies darauf hin, dass im nüchternen Zustande fast ebenso energisch als sonst die Temperatur durch Wärmeproduction regulirt wird. Dieses merkwürdige Verhältniss folgt aus Versuch I bis X. Denn hier wurde jedesmal der Versuch bei "hoher" Temperatur Morgens, nachdem die Thiere gefressen hatten, gemacht; der "Kälteversuch» aber Nachmittags, ohne dass die Thiere etwas zu fressen erhielten. In diesem nüchternen Zustand regulirten sie also durch Aufnahme fast ebenso grosser Sauerstoffquantitäten, als ob sie nicht nüchtern wären; aber der Zuwachs an Kohlensäure war bei der Abkühlung viel kleiner als im nüchternen Zustande.

VII. Da die Sauerstoffabsorption den Anfang, die Kohlensäurebildung das Ende der physiologischen Stoffmetamorphose bezeichnet und beide bei der Wärmeregulation ganz gleichzeitig und in demselben Verhältniss wachsen und abnehmen, da also zwischen Anfang und Ende des Stofiwechsels eine constante mathematische Beziehung existirt (wie unsere Versuche beweisen), so kann man nicht annehmen, dass der intermediäre Stofiwechsel nicht in gleichem sinne und nach demselben Gesetz sich ändere. Es ist also in diesem Falle unzweifelhaft Sauerstoffverbrauch und Kohlensäurebildung - wie vom Stickstoff abgesehen nach Pflüger wahrscheinlich immer ein Maass des Stoffwechsels, die Wärmeproduction dem Sauerstoffverbrauch und der Kohlensäurebildung proportional.

VIII. An den Meerschweinchen ist, wenn sie in der Kälte sitzen und die colossale Steigerung des Stoffwechsels bei ihnen anhebt, gar nichts Auffallendes zu bemerken. Sie sitzen vielmehr ganz ruhig, so wie sonst.

IX. Die durch Abkühlung bedingte Steigerung des Stoffwechsels findet keineswegs nur in den ersten Zeiten nach dem Sinken der Temperatur der Luft statt, sondern wächst sogar zuweilen (s. Tabelle) 
im Laufe von Stunden so bedeutend, dass die innere Körpertemperatur des Thieres sehr beträchtlich zunimmt.

$\mathrm{X}$. Weil mit zunehmender Körpergrösse die Oberläche in quadrischem, der Körperinhalt in cubischem Verhältniss wächst, versteht es sich von selbst, dass grosse Geschöpfe bei gleicher Temperaturerniedrigung der Luft eine relativ kleinere Steigerung ihres Stoffwechsels bedürfen. Dass ausserdem bei verweichlichten Stubenmenschen die Temperatur regulirende Wärmeproduction oft sehr schwach sein mag, soll nicht bestritten werden. - Gleichwohl handelt es sich hier um eine ganz gewaltige, höchst zweckmässige Einrichtung der Natur zur Sicherung der Existenz.

XI. Die Meerschweinchen exhaliren während der Verdauung Sumpfgas und Wasserstoff, im nüchternen Zustand kaum oder gar nicht. Das Meerschweinchen exhalirt pro Kilo und Stunde 24.6 Cc. brennbares Gas, welches im Mittel besteht aus $21.1 \mathrm{Cc}$. Sumpfgas und $3.5 \mathrm{Cc}$. Wasserstoff. (Alles bezogen auf $0^{0} \mathrm{C}$. u. $0^{\mathrm{m}} .76 \mathrm{Hg}$.)

XII. Die Meerschweinchen absorbiren weder Stickstoff noch exhaliren sie ihn. Da ich indessen nur ein geringes thatsächliches Material zur Entscheidung dieser fundamental wichtigen Frage besitze, und da hierfür eine grössere Zahl von böchst zeitraubenden und sehr subtilen und complicirten Gasanalysen nothwendig sind, so möchte ich mich mit Bezug auf Punkt XII nicht mit apodiktischer Sicherheit aussprechen. 


\begin{tabular}{|c|c|c|c|c|c|c|c|c|c|c|c|c|c|c|c|c|}
\hline $\begin{array}{c}\text { Nunmer } \\
\text { des } \\
\text { Yersudes: }\end{array}$ & Thierart. & & $\begin{array}{l}\text { Thier. } \\
\text { ewricht } \\
\text { in } \\
\text { ramin, }\end{array}$ & $\begin{array}{l}\text { Dave } \\
\text { Pers } \\
\text { Stur. } \\
\text { den. }\end{array}$ & $\begin{array}{c}\text { Minh } \\
\text { ten. }\end{array}$ & $\begin{array}{c}\text { Rect } \\
\text { tempe } \\
\text { vor dent } \\
\text { Tersuch }\end{array}$ & 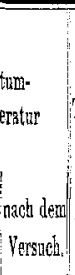 & 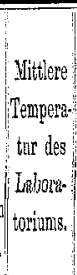 & 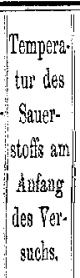 & 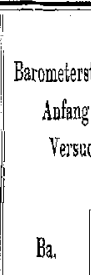 & $\begin{array}{l}\text { tand am } \\
\text { des } \\
\text { case. }\end{array}$ & $\begin{array}{l}\text { Barometers } \\
\text { Ende } \\
\text { Versu }\end{array}$ & tand am & \multicolumn{3}{|c|}{ 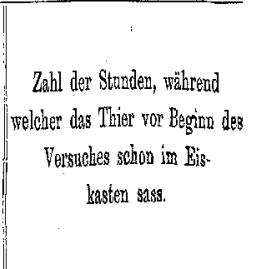 } \\
\hline I. & Heerschreinchen & & 4.1 & 3 & 14 & 38,000 & $38.20 \mathrm{C}$ & $15.90 \mathrm{C}$ & 15.500 & $0^{0 .} .791$ & $14.5^{\circ}$ & 0.7571 & $16.2^{0}$ & & & \\
\hline Il. & $y^{\circ}$ & 1 & 449 & 2 & 25 & 37.0 & 37.1 & 16.8 & 17,0 & 00.766 & $16.0^{\circ}$ & 60.7566 & 16.70 & & titunden & \\
\hline III. & $y$ & $1 \|$ & 415 & 3 & 35 & 37.0 & 37.9 & 16.8 & 16.5 & $0 \times 1.7564$ & $15.5^{0}$ & $0^{2 n}, 7564$ & $1.55^{0}$ & & & \\
\hline IV. & $\sharp$ & 1 & 408 & 2 & 21 & 37.6 & 38.0 & $16 . j$ & 17,2 & 0 . .568 & $16.5^{\circ}$ & $0^{m} .7668$ & $16.0^{0}$ & $21 / 8$ & " & \\
\hline V. & 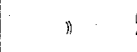 & 2 & 382 & 4 & 12 & 37.1 & 37.9 & 18.8 . & 18.0 & $0^{20.7426}$ & $17.6^{0}$ & $0=7420$ & $16.8^{\prime \prime}$ & & & \\
\hline Pl & B & $2: 3$ & 379 & 2 & 33 & 37.4 & 38.8 & 18.2 & 19.2 & $\mathrm{om}^{\mathrm{m}} .84 \mathrm{~B}^{\mathrm{B}}$ & 1879 & $\ln ^{20} .7416$ & 18.78 & 2 & & \\
\hline WII. & ) & 2 & 400 & 2 & 00 & 38.5 & 38.0 & 18.4 & 18.4 & $90.75 \pm$ & 18.20 & $020.798 \%$ & 18.29 & & & \\
\hline MIII. & $n$ & 2 & $390^{\circ}$ & 3 & 29. & 38.5 & $38.0^{\circ}$ & 18.7 & 19,3 & $0^{04} .756$ & $18.0^{\circ 0}$ & $0^{14} .756$ & $18.4^{\circ}$ & 3 & 2 & \\
\hline $\mathrm{IX}$ & 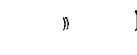 & 1 & 400 & 3 & 00 & 37.1 & 37,0 & 16.2 & 16.0 & 020.7394 & $15.4^{0}$ & (0m.7.731 & $16,2^{\circ}$ & & & \\
\hline$X_{1}$ & s & 1 & 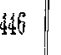 & 2 & 81 & 37.0 & 38.5 & 10.3 & 16.5 & 00.703 & $36.1^{\circ}$ & 008.9020 & 15.80 & 2 & y & \\
\hline XI. & n & 32 & 278 & $\dot{j}$ & 18 & 37.6 & 37.5 & 22.3 & 21.6 & $0^{\min .768}$ & $19.6^{\circ}$ & 00.768 & $19.9^{9}$ & & & \\
\hline XII. & ) & 32 & 278 & 3 & 30 & 36.2 & 36.3 & 21.2 & 20.5 & 0101.7648 & $20.4^{8}$ & 00.7648 & $20.4^{0}$ & $1^{1 / 2}$ & $\#$ & \\
\hline XIII. & 3 & 3 & 924 & 6 & 8 & 36.0 & 36.2 & 21.4 & 20.7 & $04.7645^{\circ}$ & $20.6^{9}$ & $0^{10.7624}$ & 21.90 & & & \\
\hline $\mathrm{XIV}$. & 》 & $4: 3$ & 304 & 3 & 6 & 37.4 & 37.0 & 22.1 & 22,2 & 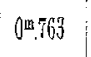 & $21.6^{6}$ & 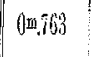 & $21.6^{\circ}$ & 2 & " & dem Yersitizh \\
\hline$X Y$. & $y$ & 4 & 292 & 4 & $4 j$ & 37.6 & 37.0 & 20.6 & 20.3 & 0 ד.7.9.68 & 19.80 & $0^{50.7} .7588$ & $19.8^{\circ}$ & & & \\
\hline XVI. & B & 4 & 284 & 3 & 21 & 37.0 & 37.3 & 19.4 & 19.3 & $0^{x} .7600$ & $19,0^{\circ}$ & $0^{m}, 7698$ & 19.80 & 2 & 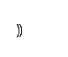 & \\
\hline XVLI. & $"$ & 4. $?$ & 999 & 2 & 12 & 37.5 & 37.1 & 20.4 & 19.8 & $0^{20} .7840$ & $19.8^{\circ}$ & 10.764 & 19.80 & 8 & : & $\begin{array}{l}\text { (Das Thier } \\
\text { ans toufang }\end{array}$ \\
\hline XVIII, & $y$ & 4 & 293 & 3 & 22 & 37.7 & 37.0 & 20.8 & 20.7 & (0. $76 \pm 0$ & $20,0^{\circ}$ & $0^{m} .7622$ & $20.6^{\circ}$ & 2 & $B$ & $\begin{array}{l}\text { Tersuciles } \ Y \text { YII } \\
\text { sehr untulig. }\end{array}$ \\
\hline XIX & $B$ & 43 & 300 & 4 & 41 & 88,0 & 37.7 & 22.7 & 20.4 & $0^{x} .7620^{\circ}$ & $20.00^{\circ}$ & $0^{m} .7605$ & $20.0^{\circ}$ & & & $\begin{array}{l}\text { Der Versuca } \\
\text { wirl dessalt }\end{array}$ \\
\hline $\mathrm{X}$ & ) & 4 & 297 & 6 & 11 & 37.0 & 37.8 & 21.1 & 21.0 & $00.7600^{\circ}$ & 20.00 & 000.7605 & 20.00 & 2 & ) & \\
\hline
\end{tabular}




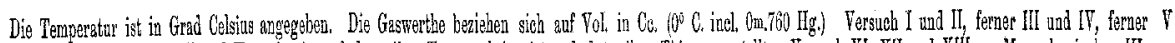

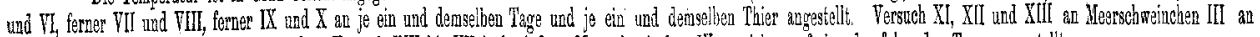
drei euf einander folgenden Tagen angestellt, Tersuch XIV bis XX inde sind an Meerschwejuchen IV an sieben suf einander folgenden Tagen angestellt.

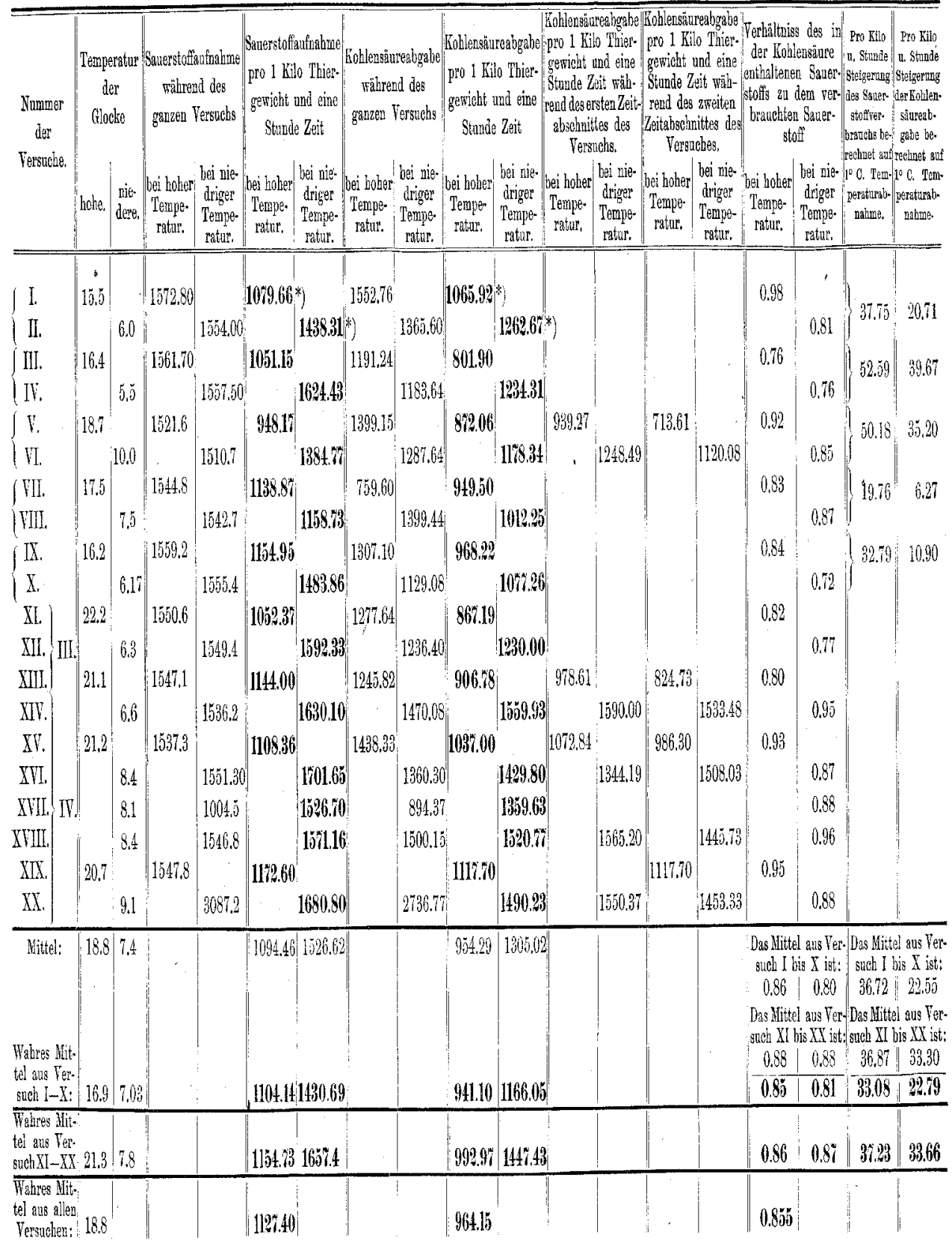

*) Dhese bier stehendea Werthe sind die nicht conriginten Werthe, wahrend in Protokoll (p. 106-108) die comiginten Werthe aufgezeichenet sind. 


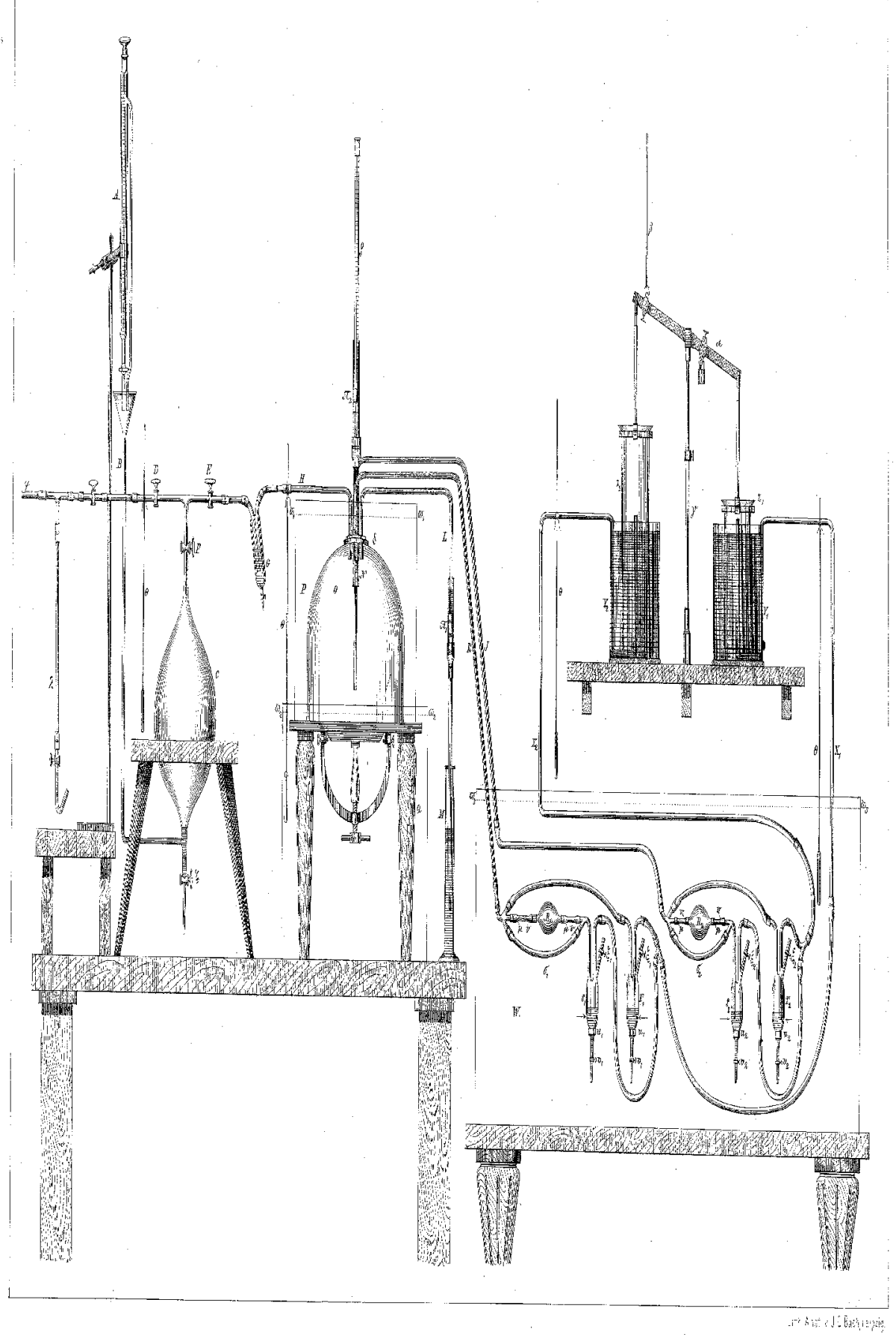

